\title{
Analyzing Image Structure by Multidimensional Frequency Modulation
}

\author{
Marios S. Pattichis, Senior Member, IEEE, and Alan C. Bovik, Fellow, IEEE
}

\begin{abstract}
We develop a mathematical framework for quantifying and understanding multidimensional frequency modulations in digital images. We begin with the widely accepted definition of the instantaneous frequency vector (IF) as the gradient of the phase and define the instantaneous frequency gradient tensor (IFGT) as the tensor of component derivatives of the IF vector. Frequency modulation bounds are derived and interpreted in terms of the eigendecomposition of the IFGT. Using the IFGT, we derive the ordinary differential equations (ODEs) that describe image flowlines. We study the diagonalization of the ODEs of multidimensional frequency modulation on the IFGT eigenvector coordinate system and suggest that separable transforms can be computed along these coordinates. We illustrate these new methods of image pattern analysis on textured and fingerprint images. We envision that this work will find value in applications involving the analysis of image textures that are nonstationary yet exhibit local regularity. Examples of such textures abound in nature.
\end{abstract}

Index Terms-Theory and models, image processing and computer vision, image models.

\section{INTRODUCTION}

$\mathrm{N}$ ONSTATIONARY texture analysis remains an important and unresolved research problem in image analysis. One of the most challenging aspects is the development of numerical measures that can capture and quantify both the texture patterns as well as information-bearing spatial variations in the patterns. An important tool that has been developed for this purpose is the Image Modulation model, also termed the Amplitude-Modulation Frequency-Modulation (AM-FM) image model, which models nonstationary image content using an AM-FM expansion.

By way of example, consider the two images in Fig. 1. The Fig. 1a shows a piece of a fingerprint, while Fig. 1b shows a woodgrain. Both images are excellent examples of emergent AM-FM patterns.

Nonstationarity in the fingerprint image manifests as variations in both the ridge orientations and in the ridge spacings. In the core region of the fingerprint-the uppermiddle region of Fig. 1a-the ridges are closely packed. Away from the core region, the spacing between the ridges increases. There is also significant variation in the ridge orientation throughout the image.

The nonstationarities present in the woodgrain image of Fig. $1 b$ are significantly more variable than those in the fingerprint. There are clear breaks and discontinuities in the grain. There is considerable variation in the grain spacing, grain orientation, and grain width.

To reveal the underlying image structure, we propose a simple image model, where the textured pattern can be

- M.S. Pattichis is with the Department of Electrical Engineering and Computer Engineering, University of New Mexico, Albuquerque, MSC01 1100, ECE Bldg., Room: 229A, Albuquerque, NM 87131. E-mail:pattichis@ece.unm.edu.

- A.C. Bovik is with the Department of Electrical and Computer Engineering, University of Texas at Austin, 1 University Station, C0803, Austin, TX 78712. E-mail: bovik@ece.utexas.edu.

Manuscript received 23 Oct. 2005; revised 13 Apr. 2006; accepted 7 Aug. 2006; published online 18 Jan. 2007.

Recommended for acceptance by J. Weickert.

For information on obtaining reprints of this article, please send e-mail to: tpami@computer.org, and reference IEEECS Log Number TPAMI-0565-1005. Digital Object Identifier no. 10.1109/TPAMI.2007.1051. thought of as a harmonic image that is coordinate transformed into the observed texture. We consider the following approximation:

$$
I(\mathbf{x}) \approx a(\mathbf{x}) \cos \varphi(\mathbf{x}) .
$$

In (1), an image $I(\mathbf{x})$ is approximated by an AM-FM image $a(\mathbf{x}) \cos \varphi(\mathbf{x})$. The AM function $a(\mathbf{x})$ is assumed to be nonnegative, slowly varying functions which correspond to the component texture envelopes or contrasts. The phase $\varphi(\mathbf{x})$ of the FM function $\cos \varphi(\mathbf{x})$ captures the pattern variation, which is more intuitively expressed by the instantaneous frequency vector (IF)

$$
\nabla \varphi(\mathbf{x})=\left[\frac{\partial \varphi}{\partial x_{1}}(\mathbf{x}), \frac{\partial \varphi}{\partial x_{2}}(\mathbf{x})\right]^{T},
$$

where $\nabla$ is the gradient operator.

In order to utilize the model (1) to analyze an image $I(\mathbf{x})$, it is first necessary to compute the AM-FM component images. Previous papers have detailed methods for achieving relevant AM-FM demodulation, namely, computation of the amplitude function, the phase function, and/or the instantaneous frequency vector function.

More complicated textured images may also be analyzed through the model in (1), but the relationship of the single dominating component to the underlying image structure will be much more complicated. Furthermore, for more complex images, continuity, and differentiability conditions will need to be enforced for the instantaneous frequency vector estimates.

In this paper, we explore how the spatial variations of the instantaneous frequency vector can be used to characterize the underlying patterns in textured images. We develop a number of new texture feature measures and show how complex patterns reveal themselves through the new measures. Geometric interpretations are given of how the proposed methods can be used to analyze complex patterns. Our primary focus is on developing the underlying theory while exploring some basic applications. We will also review several 


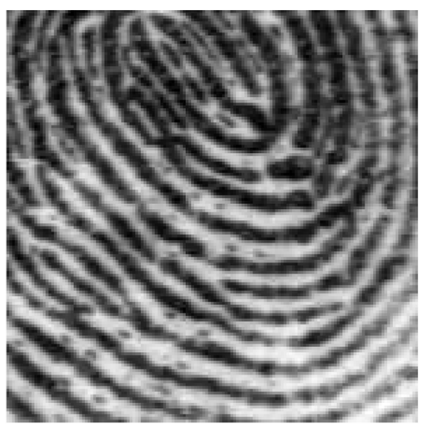

(a)

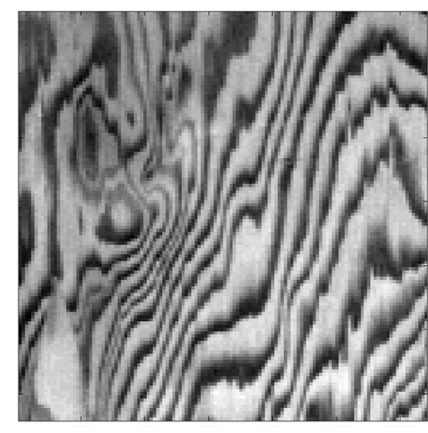

(b)
Fig. 1. Two examples of nonstationary images that can be analyzed with the proposed methods. (a) Fingerprint image. (b) Woodgrain image. The fingerprint image is the second image in the National Institute of Standards (NIST) database.

existing applications of the underlying theory and review how they can benefit from the work that we present here.

For developing the underlying theory, we note that the local variations in the instantaneous frequency vectors form flowlines that are revealed by the ordinary differential equations (ODEs) of the frequency modulation. The collection of image flowlines provides an intuitive global picture of how the underlying image patterns vary continuously through an image. We also show how a significant simplification of the ODEs can be obtained by diagonalizing them along the directions of maximum and minimum FM.

It is important to note that our local flowline approach cannot be extended at a global level. As shown by Penrose in [1, p. 438], for ridge-rich images such as fingerprints and palm-prints, there is no hope of developing global topological models assuming continuous ridge orientations. Similarly, there is no hope of imposing a globally continuous instantaneous frequency vector field on fingerprints since ridge directions are not directed [1]. In addition, in general, there is no hope of defining ridge lines globally, as constant image intensity lines [1, p. 439]. Several difficulties associated with defining ridges for general problems in image processing and computer vision are described in [2].

Early work on the importance of phase in both 1D and multidimensional signal processing was explored in [3]. In [3], Oppenheim and Lim derived conditions that allow approximate and exact reconstructions of a signal using only phase information. In [4], Fleet and Jepson developed phase-based methods for motion estimation. The authors used velocity tuned Gabor filters to show that phase-based methods can provide robust velocity estimates at a betterresolution, with subpixel accuracy.

Significant work has been done on 1D AM-FM signal analysis, particularly for application to speech signal analysis [5], [6], [7], [8], [9]. Multidimensional AM-FM demodulation methods have been studied in [10], [11], [12], [13], [14], [15], [16], [17], [18], [19], [20], [21], [22], [23], [24], [25], [26], [27], [28]. Other related research includes bounds on demodulation accuracy for discrete modulated signals [29], and Cramer-Rao bounds on AM-FM estimates [30].

AM-FM models have been used in many image pattern analysis applications, including shape from shading [31], image interpolation [32], fingerprint classification [33], image retrieval in digital libraries [34], image segmentation [35], [36], [37], and video segmentation [38].
The paper is organized as follows: Section 2 summarizes methods for 2D AM-FM demodulation. Section 3 provides a framework for understanding and interpreting multidimensional frequency modulation in images in terms of the instantaneous frequency gradient tensor (the Hessian of the phase). Section 4 derives ordinary differential equations (ODEs) that describe image flowlines, which result from solving the ODEs associated with the instantaneous frequency vectors. Expressions are also derived for the phase and the instantaneous frequency vectors. Section 5 presents geometric conditions for diagonalizing the ODEs along the eigenvector directions of the instantaneous frequency gradient tensor. Results on real patterned images are presented in Section 6, and concluding remarks are given in Section 7.

\section{Methods for Image Demodulation}

We summarize methods for demodulating multidimensional AM-FM images. A large number of 1D and 2D AMFM demodulation algorithms have been developed. The best-known of these methods are based either on the Teager-Kaiser (T-K) Operator or on the analytic signal.

\subsection{AM-FM Demodulation Using Teager-Kaiser Operators}

Given an image $I(\mathbf{x})$, the Teager-Kaiser energy operator is defined as:

$$
\Psi_{\mathrm{c}}\{I\}(\mathbf{x}) \equiv\|\nabla I(\mathbf{x})\|^{2}-I(\mathbf{x}) \nabla^{2} I(\mathbf{x}),
$$

where $\nabla^{2}=\partial^{2} / \partial x_{1}^{2}+\partial^{2} / \partial x_{2}^{2}$ is the Laplacian operator. The $\mathrm{AM}$ and FM estimates are:

$$
\begin{aligned}
& \hat{\phi}_{i}=\sqrt{\Psi_{\mathrm{c}}\left\{\frac{\partial I}{\partial x_{i}}\right\} / \Psi_{\mathrm{c}}\{I\}}, \quad i=1,2 \\
& \hat{a}=\Psi_{\mathrm{c}}\{I\} / \sqrt{\Psi_{\mathrm{c}}\left\{\frac{\partial I}{\partial x_{1}}\right\}+\Psi_{\mathrm{c}}\left\{\frac{\partial I}{\partial x_{2}}\right\}} .
\end{aligned}
$$

Note that the equations given by (4) only estimate the magnitude of the components of the instantaneous frequency vector. One method of estimating the correct signs is to form local, single harmonic images with the two possible spatial frequencies $\left(\hat{\phi}_{x_{1}}, \pm \hat{\phi}_{x_{2}}\right)$, and compute the local input image projection on the single harmonic images. The instantaneous frequency estimate which gives the corresponding largest projection is then used.

Method (3), (4), and (5) were developed for relatively noisefree images. To extend the method to noisy, wideband images, a multichannel filterbank may be deployed, applying (3), (4), and (5) at the output of each channel filter. For our examples, we use a Gabor Filter bank at 11 orientations (uniformly sampled) with constant-Q frequency-domain spacing.

In this work, we will assume that the image to be analyzed contains a single AM-FM component. Thus, we shall utilize AM-FM estimates that correspond to the dominant oriented image structure. This is accomplished by a technique called Dominant Component Analysis (DCA) (see [17]). In DCA, the image energy estimates coming from each channel are compared at each pixel, and the maximum-energy responses used in subsequent calculations. For single AM-FM component images, an alternative to Dominant Component Analysis is to use the Gabor filter outputs to compute local spectral 
moments and then use the moments to estimate the instantaneous frequency [23], [31]. In our case, we use a filterbank to separate out the dominant AM-FM component from the possible presence of multiple components (see [16] and [12] for an alternative treatment). Recently, extensions to the Teager-Kaiser operator include the work of Larkin for uniform orientation estimation [26] and the GET operator [27].

A common feature for most AM-FM demodulation methods, including the ones based on energy operators, is that they work very well with narrow-band signals. An advantage of prefiltering with the single-sided Gabor filterbank is that it provides suitable input signals to the AM-FM demodulation algorithms for accurate estimation (but see [15] for wideband signals).

\subsection{Image Demodulation Based on the Analytic Image}

Alternatively, analytic image methods for AM-FM demodulation are based on extending the notion of the 1D analytic signal to 2D or simply to provide a Hilbert-based extension of the 1D Hilbert-based demodulation approach. Early work can be found in [17]. In [24] and [25], the authors introduce the phase quadrature transform for extending the Hilbert transform into two dimensions. In [28], Felsberg and Sommer introduced the monogenic signal, a nice extension of the analytic signal to images. The monogenic signal is a threedimensional representation that combines an image with its Riesz transform to yield a sophisticated 2D analytic signal. Some related work on image demodulation based on the analytic image includes [20], [21], [22], [28].

\section{Instantaneous Frequency Gradient Tensor}

In order to develop a general model for describing complex, nonstationary textures, we use the concept of directional instantaneous frequency. Given an FM image $\cos \phi(\mathbf{x})$, the instantaneous frequency is given in terms of the gradient of the phase $\nabla \phi(\mathbf{x})=\left[\frac{\partial \phi}{\partial x_{1}}, \frac{\partial \phi}{\partial x_{2}}\right]^{T}$. The instantaneous frequency vector $\nabla \phi(\mathbf{x})$ is always orthogonal to the equi-intensity lines of both the phase $\phi(\mathbf{x})=c$ and of the original image $\cos \phi(\mathbf{x})=c$. The magnitude of the instantaneous frequency is

$$
\sqrt{\left(\frac{\partial \phi}{\partial x_{1}}\right)^{2}+\left(\frac{\partial \phi}{\partial x_{2}}\right)^{2}} .
$$

In what follows, we define the instantaneous frequency gradient tensor (IFGT) as the Hessian of the phase or, equivalently, in terms of the component derivatives of the instantaneous frequency vector

$$
\begin{aligned}
\mathbf{F}\left(x_{1}, x_{2}\right) & =\left[\begin{array}{ll}
\partial O_{1} / \partial x_{1} & \partial O_{1} / \partial x_{2} \\
\partial O_{2} / \partial x_{1} & \partial O_{2} / \partial x_{2}
\end{array}\right], \text { where } \\
\mathbf{O} & =\left[\begin{array}{l}
O_{1} \\
O_{2}
\end{array}\right]=\left[\begin{array}{l}
\partial \phi / \partial x_{1} \\
\partial \phi / \partial x_{2}
\end{array}\right] .
\end{aligned}
$$

Note that the instantaneous frequency gradient tensor is real and symmetric with real eigenvalues and orthonormal eigenvectors [39]: $\mathbf{F}=\mathbf{E} \boldsymbol{\Lambda} \mathbf{E}^{T}$, where $\mathbf{E}$ is the eigenvector matrix. We note that $\mathbf{E}$ is an orthogonal matrix with unit columns. It may thus be considered as a rotation matrix. The eigen decomposition reveals the essential, local characteristics of the multidimensional frequency modulation. As we describe in Lemma 1, the instantaneous frequency undergoes its greatest change in the spatial direction of the dominant eigenvector direction, corresponding to the largest eigenvalue. Furthermore, the minimum change occurs in the spatial direction that is orthogonal to the dominant eigenvector. Later, we will find that it is useful to analyze the eigenvector directions at each image pixel.

In the analysis that follows, we assume the usual matrix norm defined for a matrix $\mathbf{F}$ using $\|\mathbf{F}\| \equiv \max _{\mathbf{x} \neq \mathbf{0}}\|\mathbf{F x}\| /\|\mathbf{x}\|$, where: $\|\mathbf{x}\| \equiv \sqrt{\mathbf{x}^{T} \mathbf{x}}$.

Lemma 1 (Frequency modulation bounds). The differential increase in the magnitude of the instantaneous frequency vector is always bounded as:

$$
\left|\lambda_{2}\right||| d \mathbf{x}|| \leq|| d \mathbf{O}\left\|\leq\left|\lambda_{1}\right||| d \mathbf{x}\right\|, \quad\left|\lambda_{1}\right| \geq\left|\lambda_{2}\right| .
$$

The upper bound is achieved for the first eigenvector: $d \mathbf{x}=c\left[e_{1,1}, e_{1,2}\right]^{T}$, where $c$ is a scalar constant. The lower bound is achieved for the second eigenvector: $d \mathbf{x}=c\left[e_{2,1}, e_{2,2}\right]^{T}$. Clearly, when $\lambda_{1}=\lambda_{2}$, then $\|d \mathbf{O}\|=\left|\lambda_{1}\right| \| d \mathbf{x}||$.

Proof. See Appendix A.

From (7), the maximum change in the instantaneous frequency vector magnitude is in the eigenvector direction corresponding to the largest magnitude eigenvalue. By contrast, the minimum change in the instantaneous frequency vector magnitude is in the eigenvector direction corresponding to the smallest magnitude eigenvalue.

We can use the IFGT to understand the local image phase structure. Expand $\phi(\mathbf{x})$ into the Taylor series expansion about $\mathrm{x}_{0}$ [39, p. 327]:

$$
\begin{aligned}
\phi(\mathbf{x}) \approx & \phi\left(\mathbf{x}_{0}\right)+\left(\mathbf{x}-\mathbf{x}_{0}\right)^{T} \nabla \phi\left(\mathbf{x}_{0}\right) \\
& +\frac{1}{2}\left(\mathbf{x}-\mathbf{x}_{0}\right)^{T} \mathbf{F}\left(\mathbf{x}_{0}\right)\left(\mathbf{x x}_{0}\right),
\end{aligned}
$$

where the approximation is of order $\left\|\mathbf{x}-\mathbf{x}_{0}\right\|^{3}$. Equation (8) provides a quadratic, local chirp-like approximation to the underlying texture patterns. The coefficients of the quadratic terms are the matrix components of the IFGT.

The complex FM image may be thought of as a local product of two 1D signals, defined along the eigenvector directions. To show this, for $x_{0}$ given, define the eigenvector coordinates in terms of the eigenvector matrix $\mathbf{E}^{T}$

$$
\mathbf{z}(\mathbf{x})=\mathbf{E}^{T}(\mathbf{x})\left(\mathbf{x}-\mathbf{x}_{0}\right)
$$

on the rectangular Cartesian coordinates $\mathrm{x}$. We will use over a symbol to denote that it is defined on the eigenvector coordinate system. Thus, $\tilde{\phi}(\mathbf{z}), \tilde{\mathbf{O}}(\mathbf{z})$, and $\tilde{\mathbf{F}}(\mathbf{z})$ denote the phase, instantaneous-frequency, and IFGT defined on the eigenvector coordinate system. Around each point in the image, the phase is separable, meaning that it can be expressed as a sum of two one-dimensional functions. To see this, let $\mathbf{z}_{0}=\left[a_{1}, a_{2}\right]^{T}$, and note that

$$
\tilde{\phi}(\mathbf{z}) \approx \tilde{\phi}\left(a_{1}, a_{2}\right)+\tilde{\phi}_{1}\left(z_{1}\right)+\tilde{\phi}_{2}\left(z_{2}\right),
$$

where:

$$
\tilde{\phi}_{i}\left(z_{i}\right)=\frac{\partial \tilde{\phi}}{\partial z_{i}}\left(a_{1}, a_{2}\right)\left(z_{i}-a_{i}\right)+\frac{\lambda_{i}\left(a_{1}, a_{2}\right)}{2}\left(z_{i}-a_{i}\right)^{2}, \quad i=1,2,
$$


where $\lambda_{1}, \lambda_{2}$ denote the eigenvalues of $\tilde{\mathbf{F}}$. Note that the approximation holds when $\mathbf{z}$ is near the origin. In what follows, without loss of generality, we take $\mathbf{z}_{0}=[0,0]^{T}$. We also note that the complex FM function is locally approximated by a product of two 1D FM functions defined on the eigenvector coordinate directions:

$$
\exp \left[j \tilde{\phi}\left(z_{1}, z_{2}\right)\right] \approx \exp \left[j \phi_{1}\left(z_{1}\right)\right] \exp \left[j \phi_{2}\left(z_{2}\right)\right] .
$$

The result in (12) can be directly extended to any finite number of dimensions.

The approximation (12) suggests that we can locally describe multidimensional frequency modulation as a product of 1D frequency modulation processes that are defined along the eigenvector directions.

\section{The ODEs of Multidimensional Frequency MODULATION}

We now develop nonlocal methods for characterizing multidimensional frequency modulation. In an FM image, the instantaneous frequency vector remains orthogonal to the image equi-intensity lines. To track changes in intensity due to FM, we analyze the image in the direction of the instantaneous frequency vector. This is accomplished through the use of the FM ODEs.

To clarify the method, we suggest an analogy from fluid mechanics, wherein we interpret instantaneous frequency vectors as velocity vectors. We are given:

- $\mathbf{F}$ is the instantaneous frequency gradient tensor (similar to the velocity gradient tensor in fluid mechanics).

- The initial conditions: 1) an image point $\mathbf{x}_{0}$, 2) $\mathbf{O}\left(\mathbf{x}_{0}\right)$, the initial instantaneous frequency vector, and 3) $\phi\left(\mathbf{x}_{0}\right)$, the initial value of the phase function. For selecting suitable initial points $\mathbf{x}_{0}$, in the presence of FM (nonzero IFGT), we note that flowline computation amounts to gradient ascent to a stationary point of the phase.

We can think of $\mathbf{x}_{0}$ as a reference point, at which the phase function assumes a reference value $\phi\left(\mathbf{x}_{0}\right)$. At the reference point $\mathbf{x}_{0}$, we need to compute: 1$) \mathbf{x}(t)$, the image flow line, 2) $\mathbf{O}(\mathbf{x}(t))$, the instantaneous frequency vector along the flow line, and 3) $\phi(\mathbf{x}(t))$, the phase function along the trajectory.

The following theorem provides the ODEs for the instantaneous frequency and the flowlines:

Theorem 1. (General FM ODEs). Assume that the instantaneous frequency vector $\mathbf{O}(\mathbf{x})$ is continuously differentiable and tangent to a continuous flowline path $\mathbf{x}(t)$

$$
\frac{d \mathbf{x}}{d t}(t)=\mathbf{O}(\mathbf{x}(t)) .
$$

Then, the flowline is

$$
\mathbf{x}(t)=\mathbf{x}_{0}+\int_{0}^{t} \mathbf{O}(\mathbf{x}(\tau)) d \tau .
$$

Along the flowline, the instantaneous frequency is

$$
\mathbf{O}(\mathbf{x}(t))=\mathbf{O}\left(\mathbf{x}_{0}\right)+\int_{0}^{t} \mathbf{F}(\mathbf{x}(\tau)) \mathbf{O}(\mathbf{x}(\tau)) d \tau
$$

and the phase is

$$
\phi(\mathbf{x}(t))=\phi\left(\mathbf{x}_{0}\right)+\int_{0}^{t}\left\|\mathbf{O}(\mathbf{x}(\tau))^{2}\right\| d \tau .
$$

\section{Proof. See Appendix B.}

We now provide geometric interpretations of multidimensional frequency modulation along the image flowlines. We view image flowlines as trajectories that describe image flow throughout the image. To see what happens to a neighborhood of image points that follow these trajectories, consider the deformation of a small circular disc neighborhood subjected to image flow. As the disc moves along the flowline, it is deformed into a new ellipsoidal disc. The eigenvectors are in the directions of the principal axes of this ellipsoidal disc [40]. Thus, we term the eigenvectors the principal axes of frequency modulation. The eigenvalues are relatively proportional to the axes of the ellipse [39]. At any particular point in the image, the divergence of the vector measures the relative expansions in the area of the disc [41]. If the disc is of area $S$ and $t$ denotes the time along the trajectory, the relative rate of change of $S$ comes from

$$
\begin{aligned}
\operatorname{div} \mathbf{O}(\mathbf{x}) & =\frac{\partial O_{1}}{\partial x_{1}}+\frac{\partial O_{2}}{\partial x_{2}} \\
& =\operatorname{trace}\{\mathbf{F}\} \\
& =\lambda_{1}+\lambda_{2} . \\
& =\frac{1}{S} \frac{d S}{d t} .
\end{aligned}
$$

The curl of the instantaneous frequency is

$$
\nabla \mathbf{O}(\mathbf{x})=\left(\frac{\partial O_{2}}{\partial x_{1}}-\frac{\partial O_{1}}{\partial x_{2}}\right) \overrightarrow{\mathbf{a}}_{3},
$$

where $\overrightarrow{\mathbf{a}}_{3}$ denotes the unit vector along the axis perpendicular to the image-plane. From (21), assuming that we have continuity up to the second derivatives, we note that $\partial O_{2} / \partial x_{1}=\partial^{2} \phi / \partial x_{2} \partial x_{1}=\partial^{2} \phi / \partial x_{1} \partial x_{2}=\partial O_{1} / \partial x_{2}$ which implies that the curl of the instantaneous frequency will be zero, except at points of discontinuity where the second partials are not equal: $\partial^{2} \phi / \partial x_{2} \partial x_{1} \neq \partial^{2} \phi / \partial x_{1} \partial x_{2}$. At singular points in the image, such as object boundaries, the instantaneous frequency vector field will be discontinuous, and the curl will assume significant, nonzero values. The curl is associated with strong rotations in the direction of the instantaneous frequency vector field. For flowline computation, we take the average of the mixed-partial estimates $\left(\partial \widehat{O_{1} / \partial} x_{2}+\partial \widehat{O_{2} / \partial} x_{1}\right) / 2$ and set both estimates to this average value. This enforces the required symmetry of the IFGT.

\subsection{Flowline Parameters for Constant IFGT}

As a useful and instructive example, we shall compute the flowline $\mathbf{x}(t)$, the instantaneous frequency $\mathbf{O}(\mathbf{x}(t))$, and the phase $\phi(\mathbf{x}(t))$ for the special case of a constant, invertible IFGT. We will also solve the ODEs for this case.

For constant $\mathbf{F}$

$$
\frac{d \mathbf{O}}{d t}(\mathbf{x}(t))=\mathbf{F} \mathbf{O}(\mathbf{x}(t))
$$


which yields the following simplified expression for the instantaneous frequency vector along the flowline:

$$
\mathbf{O}(\mathbf{x}(t))=\exp (\mathbf{F} t) \mathbf{O}\left(\mathbf{x}_{0}\right) \text {. }
$$

On the flowline, substitute (23) into (14) and integrate to get:

$$
\left.\mathbf{x}(t)=\mathbf{x}_{0}+\mathbf{F}^{-1}[\exp f \mathbf{F} t)-I\right] \mathbf{O}\left(\mathbf{x}_{0}\right),
$$

that can also be expressed as:

$$
\mathbf{x}(t)=\mathbf{x}_{0}+\mathbf{F}^{-1}\left[\mathbf{O}(\mathbf{x}(t))-\mathbf{O}\left(\mathbf{x}_{0}\right)\right] .
$$

To compute the phase along the flowline, begin with the general expression given in (16) in Appendix B, substitute (23), and use the symmetry of $\mathbf{F}$ to get [42, p. 167]:

$$
\phi(\mathbf{x}(t))=\phi\left(\mathbf{x}_{0}\right)+\frac{1}{2} \mathbf{O}^{T}\left(\mathbf{x}_{0}\right) \mathbf{F}^{-1}[\exp (2 \mathbf{F} t)-I] \mathbf{O}\left(\mathbf{x}_{0}\right) .
$$

To interpret (26), we note from (16) that the phase will always increase along the flowline. This is to be expected since flowlines follow the direction of steepest ascent: $\nabla \phi(\mathbf{x})$. We know from (23) that $\|\mathbf{O}(x(t))\| \rightarrow 0$ implying that $\|\nabla \phi(x(t))\| \rightarrow 0$ as $t \rightarrow \infty$ and, thus, the terminal point for this case is a stationary point for the phase. Since the Hessian of the phase $\mathbf{F}$ has negative eigenvalues, we also conclude that this point is a local maximum.

\subsection{ODEs in the Eigenvector Coordinate System}

Assuming constant IFGT, separable solutions to (14), (15), and (16) are obtained in the eigenvector coordinate system. Separability greatly simplifies the computation by reducing a two-dimensional problem to two one-dimensional problems. Along the eigenvectors of the IFGT, it is easy to show that the instantaneous frequency vector is given by

$$
\nabla \tilde{\phi}(\mathbf{z}(t))=\left[\begin{array}{l}
\exp \left(\tilde{\lambda}_{1} t\right)\left(\partial \tilde{\phi} / \partial z_{1}\right)\left(\mathbf{z}_{0}\right) \\
\exp \left(\tilde{\lambda}_{2} t\right)\left(\partial \tilde{\phi} / \partial z_{2}\right)\left(\mathbf{z}_{0}\right)
\end{array}\right] .
$$

To obtain expressions for the flowlines and the phase, substitute (27) into (25), (26) to get: which gives

$$
\mathbf{z}(t)=\mathbf{z}_{0}+\left[\begin{array}{l}
\left(1 / \tilde{\lambda}_{1}\right)\left(\exp \left(\tilde{\lambda}_{1} t\right)-1\right)\left(\partial \tilde{\phi} / \partial z_{1}\right)\left(\mathbf{z}_{0}\right) \\
\left(1 / \tilde{\lambda}_{2}\right)\left(\exp \left(\tilde{\lambda}_{2} t\right)-1\right)\left(\partial \tilde{\phi} / \partial z_{2}\right)\left(\mathbf{z}_{0}\right)
\end{array}\right]
$$

and

$$
\begin{aligned}
\tilde{\phi}[\mathbf{z}(t)]=\tilde{\phi}\left(\mathbf{z}_{0}\right) & +\frac{1}{2 \tilde{\lambda}_{1}}\left[\exp \left(2 \tilde{\lambda}_{1} t\right)-1\right]\left[\frac{\partial \tilde{\phi}}{\partial z_{1}}\left(\mathbf{z}_{0}\right)\right]^{2} \\
& +\frac{1}{2 \tilde{\lambda}_{2}}\left[\exp \left(2 \tilde{\lambda}_{2} t\right)-1\right]\left[\frac{\partial \tilde{\phi}}{\partial z_{2}}\left(\mathbf{z}_{0}\right)\right]^{2} .
\end{aligned}
$$

\subsection{Bifurcations of Multidimensional Frequency Modulation}

Understanding the topology of the instantaneous frequency (IF) vector field is extremely interesting, since non-topological variations in the IF vector field can be modeled as coordinate transformations of the underlying image coordinate system, while topological changes cannot be introduced through changes in the image coordinate system. The goal of the following discussion is to understand the kinds of real-life image patterns that can be explained by our differentiable model of frequency modulation flow. At points where the
IF vector is zero, we have that the phase is stationary. We note that the topology of the IF vector field can only be changed at these stationary points. We thus examine the structure of an FM image around the neighborhood of such points, in order to understand the types of topological points in real-world images that can be explained from such an FM model. Beyond the topological points of the IF field, we are also interested at termination points, where image flowlines terminate. As we shall see below, bifurcation points of multidimensional FM include the topological points of the IF field.

In the terminology of nonlinear dynamics, a bifurcation point occurs where the solution to a dynamical system is stationary: $d \mathbf{O}(\mathbf{x}(t)) / d t=0$. Begin with the ODE for the instantaneous frequency vector

$$
\frac{d \mathbf{O}}{d t}(\mathbf{x}(t))=\mathbf{F}(\mathbf{x}(t)) \mathbf{O}(\mathbf{x}(t)) .
$$

Clearly, if $\mathbf{O}$ is constant, $\mathbf{F}$ is the zero matrix and $d \mathbf{O} / d t=0$. We will not examine this trivial case.

First, we ask: What is the physical meaning of a bifurcation point? At bifurcation points the RHS of (30) is zero. From the local Taylor series expansion in the eigenvector coordinate system, this means

$$
\left[\begin{array}{l}
0 \\
0
\end{array}\right]=\left[\begin{array}{l}
\lambda_{1} O_{1} \\
\lambda_{2} O_{2}
\end{array}\right] \text {. }
$$

The bifurcation points are evident from (31). Next, we will list the possible instances of bifurcation points. We will use an ongoing example to help visualize the bifurcation points and their effects on local image structure. Fig. 2 depicts the synthetic image $I(x, y)=$ $\cos \phi(x, y)$ with $\phi(x, y)=x^{3}+y^{3}-9 x-9 y$. The instantaneous frequency is $\nabla \phi(x, y)=\left(3 x^{2}-9,3 y^{2}-9\right)$ and the IFGT is

$$
\mathbf{F}=\left[\begin{array}{cc}
6 x & 0 \\
0 & 6 y
\end{array}\right]
$$

The possible bifurcation points are:

- $\lambda_{1}=\lambda_{2}=0$. In this case, the IFGT is zero, namely, the instantaneous frequency is constant and the local pattern structure looks like a sinusoid

$$
\begin{aligned}
\cos \phi\left(\mathbf{z}_{\mathbf{b}}+\delta \mathbf{z}\right)= & \cos \phi\left(\mathbf{z}_{\mathbf{b}}\right) \\
& -\nabla \phi \cdot \delta \mathbf{z} \sin \phi\left(\mathbf{z}_{\mathbf{b}}\right)+O\left(|\delta \mathbf{z}|^{3}\right) .
\end{aligned}
$$

In the example in Fig. 2, the sinusoidal structure around $(0,0)$ is clearly evident.

- $\lambda_{1}=0, O_{2}=0$. Since $\left|\lambda_{1}\right| \geq\left|\lambda_{2}\right|$, this case is identical to $\lambda_{1}=\lambda_{2}=0$, which we have just described.

- $\lambda_{2}=0, O_{1}=0$. In this case, $\partial \phi\left(z_{b}\right) / \partial z_{1}=0$, the quadratic term in the first eigenvector coordinate dominates causing the image to appear compressed along the dominant eigenvector direction. In this direction, the image appears as a quadratic

$$
\begin{aligned}
\cos \phi\left(\mathbf{z}_{\mathbf{b}}+\delta \mathbf{z}\right)= & \cos \phi\left(\mathbf{z}_{\mathbf{b}}\right)-\frac{\partial \phi}{\partial z_{2}}\left(\mathbf{z}_{\mathbf{b}}\right) \delta z_{2} \sin \phi\left(\mathbf{z}_{\mathbf{b}}\right) \\
& -\frac{1}{2} \cos \phi\left(\mathbf{z}_{\mathbf{b}}\right) \delta z_{1}^{2}+O\left(|\delta \mathbf{z}|^{3}\right) .
\end{aligned}
$$




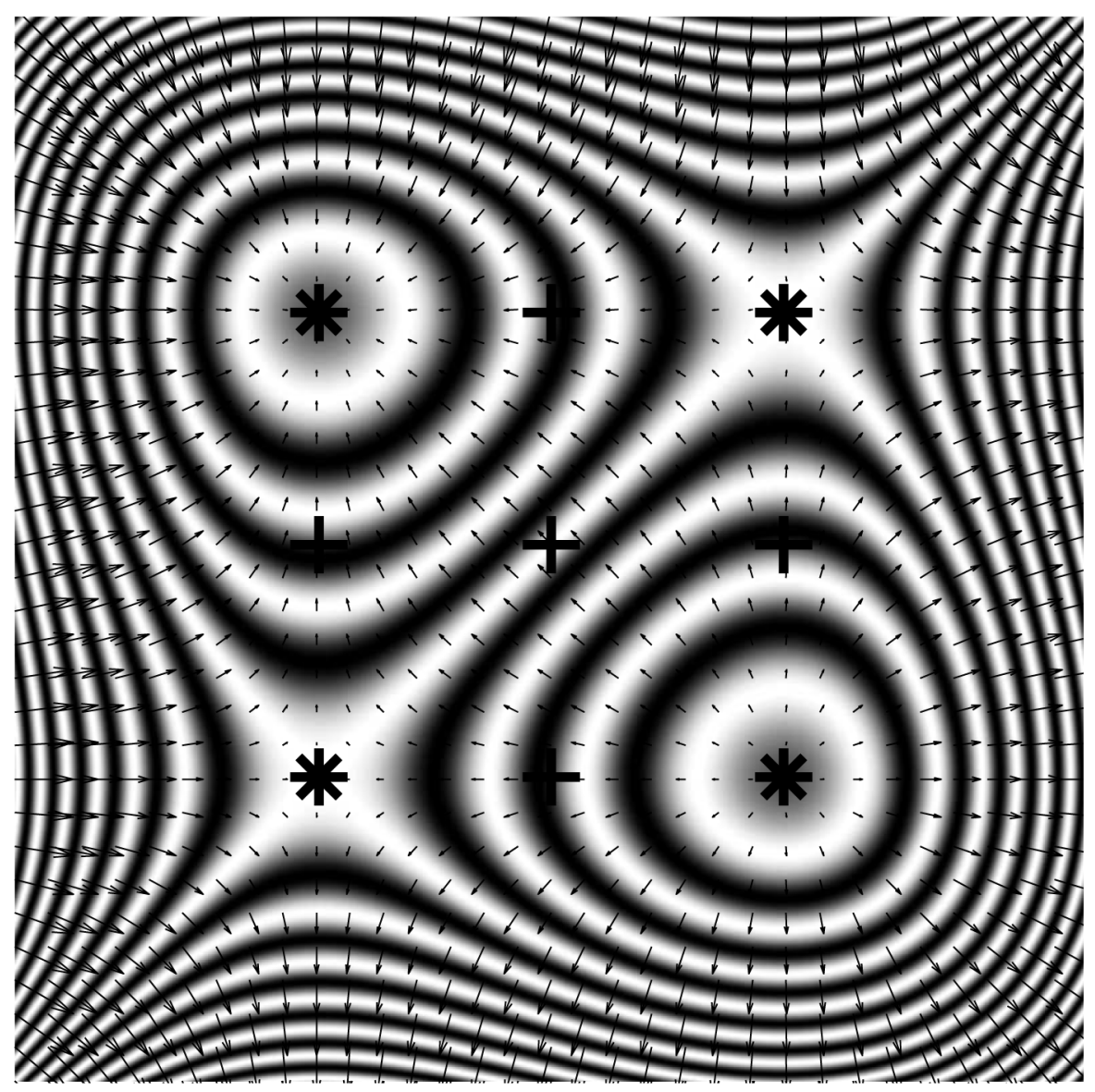

Fig. 2. The bifurcation points computed for $\phi=x^{3}+y^{3}-9 x-9 y$. The background image is $I=\cos \phi$, while the instantaneous frequency (IF) vectors are overlaid on the image. The bifurcation points are marked (see text for details). We use "+" to mark the FM bifurcation points where the IF vectors are nonzero, and "*” to mark the bifurcation points where the IF vector is zero.

In Fig. 2, $\lambda_{2}=0$ and $O_{1}=0$ at the points $(0, \pm \sqrt{3})$ and $( \pm \sqrt{3}, 0)$. The local quadratic structure is qualitatively evident at these points.

- $O_{1}=O_{2}=0$. Note that these bifurcation points of frequency modulation are also bifurcation points of phase modulation since the phase ODE is (74)

$$
\frac{d \phi}{d t}(\mathbf{x}(t))=\|\mathbf{O}(\mathbf{x}(t))\|^{2},
$$

which is zero whenever $\mathbf{O}$ is zero. There are only two types of bifurcation points in this case [43]: 1) The eigenvalues are of the same sign and 2) the eigenvalues are of different signs. We consider the cases of having two negative or two positive eigenvalues to be identical; this arises from the ambiguity between $\nabla \phi$ and $-\nabla \phi$, both of which correspond to the same FM function $\cos \phi$.

In Fig. 2, the instantaneous frequency vector becomes zero at the four points $( \pm \sqrt{3}, \pm \sqrt{3})$, at which the eigenvalues maintain the same signs as the rectangular Cartesian coordinate system points: $( \pm, \pm)$. By inspecting these four points in Fig. 2, it becomes apparent that there are only two distinct types to consider, as expected.

If the eigenvalues are of the same sign, a local, chirp-like image occurs. In Fig. 2, such a structure occurs about the bifurcation points at $(\sqrt{3}, \sqrt{3})$ and $(-\sqrt{3},-\sqrt{3})$. When the eigenvalues are of opposite sign, a saddle point occurs, with the instantaneous frequency locally increasing away from the bifurcation point.

From our discussion, it is clear that image points where the instantaneous frequency vector has a zero magnitude are the most interesting ones. In the image processing literature, points where the gradient of image intensity is zero are known as critical points [44], [45]. Here, we consider critical points as a special case of bifurcation points where the flowline computation must be terminated. We refer to [44] for the topology of such systems, but also to [46] for global methods for extracting boundaries defined from these critical points. In image regions without critical points, it is possible to model multidimensional frequency modulation in terms of a coordinate transformation of a simple sinusoid. We will demonstrate an application of this principle in Section 6.4.

\section{Diagonalization the ODEs of MULTIDIMENSIONAL FreQuency MOdULATION}

When the ODEs can be diagonalized, the complexity of analyzing multidimensional frequency modulation can be significantly reduced by using 1D differential equations in each direction. We seek general, global conditions for diagonalization that do not require a constant IFGT. Following our previous discussion, we seek such approximations in the coordinate system defined by the eigenvectors of the IFGT. 
We thus investigate the approximations

$$
\frac{d \tilde{O}_{i}(\mathbf{z}(t))}{d t} \approx \tilde{\lambda}_{i}(\mathbf{z}(t)) \tilde{O}_{i}(\mathbf{z}(t)), \quad i=1,2,
$$

which leads to the phase function being approximately separable

$$
\tilde{\phi}(\mathbf{z}) \approx \tilde{\phi}_{1}\left(z_{1}\right)+\tilde{\phi}_{2}\left(z_{2}\right) .
$$

We will derive some error bounds related to these approximations and also approximate solutions for the phase and the instantaneous frequency vectors.

First, we state two fundamental approximations which assume that the eigenvector matrix is slowly varying:

Assumption 1 (Slowly varying eigenvector approximations). Recall the definition of the eigenvector coordinate system $\mathbf{z}=\mathbf{E}^{T} \mathbf{x}$, where $\mathbf{E}$ is also a function of $\mathbf{x}$. Define the slowly varying eigenvector approximations:

$$
\frac{d \mathbf{z}}{d t} \approx \mathbf{E}^{T} \frac{d \mathbf{x}}{d t}
$$

and

$$
\frac{d^{2} \mathbf{z}}{d t^{2}} \approx \mathbf{E}^{T} \frac{d^{2} \mathbf{x}}{d t^{2}}
$$

Using $\tilde{\mathbf{O}}(\mathbf{z}(t))=d \mathbf{z} / d t$ and $\mathbf{O}(\mathbf{x}(t))=d \mathbf{x} / d t$, rewrite (36) and (37) as:

$$
\begin{gathered}
\tilde{\mathbf{O}}(\mathbf{z}(t)) \approx \mathbf{E}^{T} \mathbf{O}(\mathbf{x}(t)), \\
\frac{d \tilde{\mathbf{O}}(\mathbf{z}(t))}{d t} \approx \mathbf{E}^{T} \frac{d \mathbf{O}(\mathbf{x}(t))}{d t} .
\end{gathered}
$$

We assume that each eigenvalue changes in the direction of its corresponding eigenvector direction, as defined next.

Assumption 2. (Eigenvalue separability approximation). Let $\lambda_{1}(\mathbf{z}(t))$ and $\lambda_{2}(\mathbf{z}(t))$ denote the eigenvalues of the IFGT. Assume that the eigenvalues vary along the direction of their corresponding eigenvectors

$$
\tilde{\lambda}_{i}(\mathbf{z}(t)) \approx \tilde{\lambda}_{i}\left(z_{i}(t)\right), \quad i=1,2 .
$$

Next, we obtain intuitive error bounds on the approximations in terms of the eigenvectors. Since $\mathbf{E}$ depends on $\mathbf{F}(\mathbf{x}(t))$, it varies continuously along the flow line. In addition, note that $\mathbf{E}^{T}$ is a rotation matrix since its rows are unit eigenvectors. Thus,

$$
\mathbf{E}^{T}(\mathbf{x}(t))=\left[\begin{array}{cc}
\cos \theta(\mathbf{x}(t)) & \sin \theta(\mathbf{x}(t)) \\
-\sin \theta(\mathbf{x}(t)) & \cos \theta(\mathbf{x}(t))
\end{array}\right],
$$

which gives an expression for $d \mathbf{E}^{T} / d t$ :

$$
\frac{d \mathbf{E}^{T}}{d t}=\mathbf{U}_{1} \frac{d \theta}{d t}
$$

where $\mathbf{U}_{1}$ is a unitary matrix. The second derivative is

$$
\frac{d^{2} \mathbf{E}^{T}}{d t^{2}}=\frac{d \mathbf{U}_{1}}{d t} \frac{d \theta}{d t}+\mathbf{U}_{1} \frac{d^{2} \theta}{d t^{2}},
$$

where

$$
\frac{d \mathbf{U}_{1}}{d t}=\mathbf{U}_{2} \frac{d \theta}{d t}, \text { and } \mathbf{U}_{2} \text { is unitary. }
$$

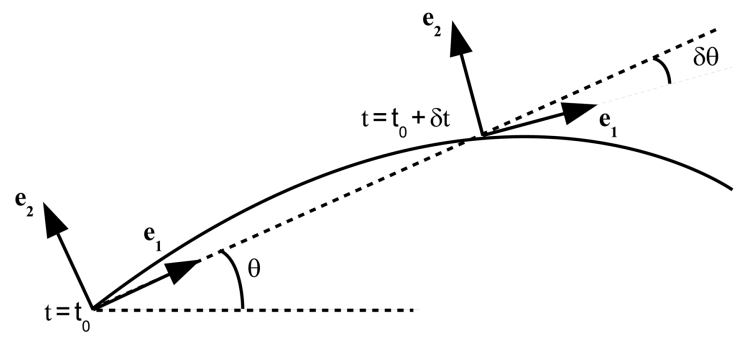

Fig. 3. The assumption of slowly varying eigenvectors. The eigenvectors $\mathbf{e}_{1}$ and $\mathbf{e}_{2}$ are carried into a new state at time $t=t_{0}+\delta t$. For slowly varying eigenvectors, the rates of rotation $\delta \theta / \delta t$ and $\delta^{2} \theta / \delta t^{2}$ are expected to be small.

Using (44), (43) may be rewritten:

$$
\frac{d^{2} \mathbf{E}^{T}}{d t^{2}}=\mathbf{U}_{2}\left(\frac{d \theta}{d t}\right)^{2}+\mathbf{U}_{1} \frac{d^{2} \theta}{d t^{2}} .
$$

We are now ready to provide an intuitive interpretation of the error in terms of the eigenvectors.

Theorem 2 (Error bounds on separability approximation).

The error in the approximation (36) is given by

$$
\begin{aligned}
\left\|\varepsilon_{1}\right\| & =\left\|\frac{d \mathbf{z}}{d t}-\mathbf{E}^{T} \frac{d \mathbf{x}}{d t}\right\| \\
& =\left|\frac{d \theta}{d t}\right|\|\mathbf{x}\|,
\end{aligned}
$$

while the error in (37) is bounded as

$$
\begin{aligned}
\left\|\varepsilon_{2}\right\| & =\left\|\frac{d^{2} \mathbf{z}}{d t^{2}}-\mathbf{E}^{T} \frac{d^{2} \mathbf{x}}{d t}\right\| \\
& \leq\left(\frac{d \theta}{d t}\right)^{2}\|\mathbf{x}\|+\left|\frac{d^{2} \theta}{d t^{2}}\right|\|\mathbf{x}\|+2\left|\frac{d \theta}{d t}\right|\|\mathbf{O}(\mathbf{x}(t))\| .
\end{aligned}
$$

Proof. The proof is given in Appendix C.

In both expressions (47) and (49), the errors are small provided that the eigenvectors rotate sufficiently slowly along the flow line, i.e., $|d \theta / d t|$ and $\left|d^{2} \theta / d t^{2}\right|$ are sufficiently small. The slowly varying assumption is demonstrated in Fig. 3.

We now show that the instantaneous frequency vector ODE is diagonalized under the slowly varying eigenvector approximation.

\subsection{Diagonalization of the Instantaneous Frequency Vector ODE}

Consider again, the instantaneous frequency ODE

$$
\frac{d \mathbf{O}(\mathbf{x}(t))}{d t}=\mathbf{F}(\mathbf{x}(t)) \mathbf{O}(\mathbf{x}(t)) .
$$

Under the slowly varying eigenvector approximation (given in (36) and (37)), (50) is diagonalized into

$$
\frac{d \tilde{O}_{i}(\mathbf{z}(t))}{d t} \approx \tilde{\lambda}_{i}(\mathbf{z}(t)) \tilde{O}_{i}(\mathbf{z}(t)), \quad i=1,2,
$$

where the equations in (51) are approximations to the flowline equations in the eigenvector coordinate system. The validity of (51) is established as follows: Multiply both sides of (50) by $\mathbf{E}^{T}$, then rewrite (50) as 


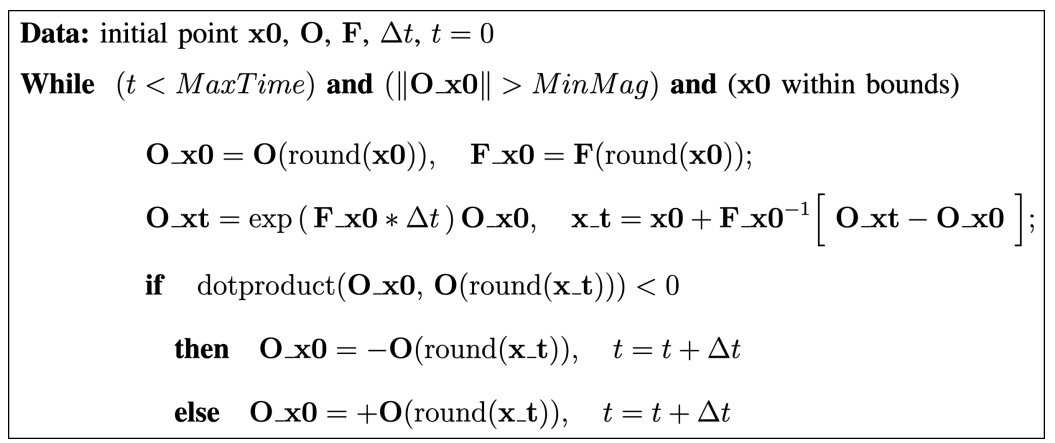

Fig. 4. Basic flowline computation algorithm. Note that round is used to round-up flowline computation to the nearest pixel. This is used to provide nearest-neighbor interpolation values for the IF and IFGT. Furthermore, the IF direction may have to be reversed to deal with sign ambiguities (see text). We refer to this as direction correction.

$$
\mathbf{E}^{T} \frac{d \mathbf{O}(\mathbf{x}(t))}{d t}=\mathbf{E}^{T}\left(\mathbf{E} \Lambda \mathbf{E}^{T}\right) \mathbf{O}(\mathbf{x}(t)) .
$$

Using (38) and (39), then yields

$$
\frac{d \tilde{\mathbf{O}}(\mathbf{z}(t))}{d t} \approx \Lambda(\mathbf{z}(t)) \tilde{\mathbf{O}}(\mathbf{z}(t)),
$$

where $\Lambda(\mathbf{z}(t))=\operatorname{diag}\left(\lambda_{1}\left(z_{1}(t)\right), \lambda_{2}\left(z_{2}(t)\right)\right)$ which establishes (51).

We are now ready to show how the phase function can be separated into two functions defined on a single eigenvector coordinate.

Lemma 2 (Phase separability in the eigenvector coordinate system). Consider an image region $S$. Assume that each interior point of $S$ can be reached from a flow line originating from the boundary of $S$. Assume that both the slowly varying eigenvector approximation and the eigenvalue separability approximation hold (Definitions 1 and 2). Then, the phase function is separable in the eigenvector coordinate system

$$
\tilde{\phi}(\mathbf{z}) \approx \tilde{\phi}_{1}\left(z_{1}\right)+\tilde{\phi}_{2}\left(z_{2}\right) .
$$

Furthermore, the instantaneous frequency components are given by

$$
\tilde{O}_{i}\left(z_{i}(t)\right) \approx a_{i} \exp \left(\int^{t} \tilde{\lambda}_{i}\left(z_{i}(\tau)\right) d \tau\right), \quad i=1,2 .
$$

Proof. See Appendix C.

\section{Computational Examples}

We present sample applications of the ODEs of multidimensional frequency modulation. In Section 6.1, we present results for flowline computation on chirp images using the proposed method and compare against three Runge-Kutta methods, to demonstrate that the proposed method is significantly more accurate. In Section 6.2, we present FM flowline computation and ridge extraction on a fingerprint image. In Section 6.3, we compute several of the proposed FM model measures from a woodgrain image, and use them to explain how they reflect the underlying image structures. In Section 6.4, we show an example of how diagonalizing the ODEs of FM can be used to reveal the underlying structure of a woodgrain image. In particular, we show how the FM ODEs can be used to model a rather complex part of the woodgrain image as a coordinatetransformed version of a simple harmonic image.

\subsection{FM Flowline Computation on Chirp Images: Comparative Examples}

In this section, we provide comparative examples with the proposed flowline computation approach and well-established Runge-Kutta methods. We present results for chirp images for which we can solve the underlying differential equations analytically.

We consider a simple chirp image with:

$$
\phi\left(x_{1}, x_{2}\right)=a\left(x_{1}-x_{c 1}\right)^{2}+b\left(x-x_{c 2}\right)^{2}, \quad a, b \geq 0 .
$$

The instantaneous frequency vector field is given by $\nabla \phi\left(x_{1}, x_{2}\right)=\left(2 a\left(x_{1}-x_{c 1}\right), 2 b\left(x_{2}-x_{c 2}\right)\right)$.

In this separable example, the autonomous equations for the flowlines become:

$$
\frac{d x_{2}}{d x_{1}}=\frac{\phi_{x_{2}}\left(x_{2}\right)}{\phi_{x_{1}}\left(x_{1}\right)}=\frac{b\left(x_{2}-x_{c 2}\right)}{a\left(x_{1}-x_{c 1}\right)} .
$$

To solve (56), fix the initial point at $\left(x_{1,0}, x_{2,0}\right)$ so that $x_{1,0}>$ $x_{c 1}$ and $x_{2,0}>x_{c 2}$ (other cases are easy to handle). We integrate (56) to get

$$
x_{2}\left(x_{1}\right)=e\left(x_{1}-x_{c 1}\right)^{b / a}+x_{c 2} \text { with } e=\frac{\left(x_{2,0}-x_{c 2}\right)}{\left(x_{1,0}-x_{c 1}\right)^{b / a}}
$$

for $x_{2} \geq x_{c 2}, x_{1} \geq x_{c 1}$. In (56), it is important to identify that $b / a$ can be any real number without being restricted to the integers. Thus, numerical methods that are based on polynomial approximations can never provide exact solutions for chirp images. The same applies for Runge-Kutta methods [47, pp. 420-424]. On the other hand, we note that (25) is exact for all real values of $a$ and $b$.

The proposed algorithm used for flowline computation is given in Fig. 4. It is based on a piecewise-constant IFGT approximation. For updating the IF and IFGT estimates, we use nearest neighbor interpolation (see Fig. 4).

We present three examples in Fig. 5. We compare our proposed method against the Runge-Kutta $(4,5)$ formula method (ode45(.), [48]), the modified Runge-Kutta $(2,3)$ pair for moderately stiff problems (ode23(.), [49]), and a stiff solver (ode23s(.), [50]). For all methods, we assume that the instantaneous frequency vectors are available at each pixel. From the results in Figs. $5 b$ and $5 d$, it is clear that all methods achieved unbiased (except possibly for a positive bias in ode23s(.)), subpixel accuracy. Furthermore, it is also clear that the proposed method is significantly more accurate, in orders of magnitude, than all the other methods. 


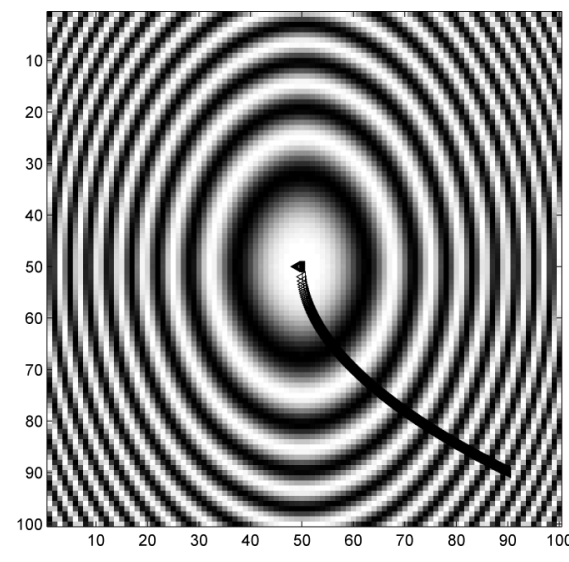

(a)

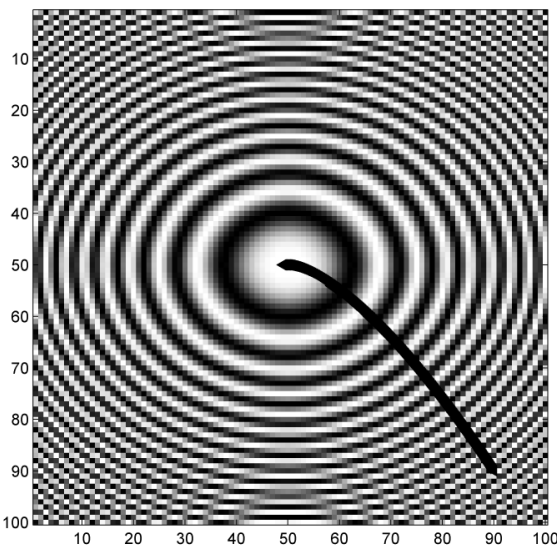

(c)

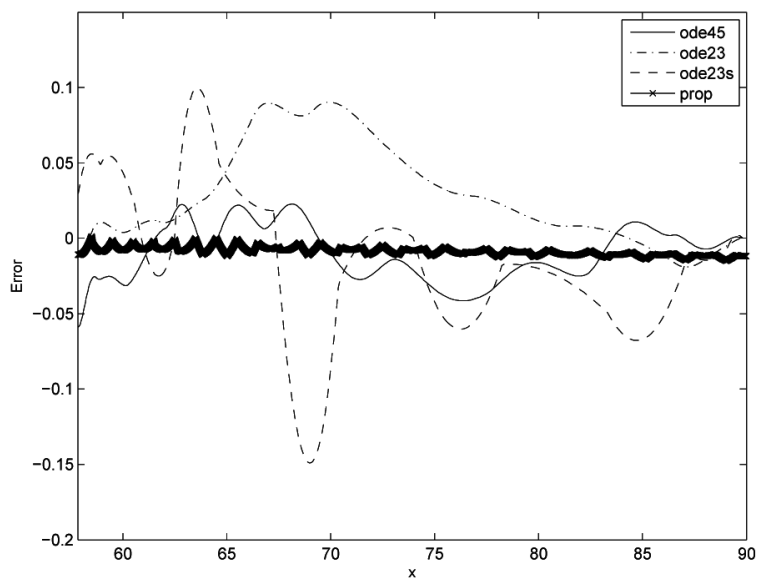

(b)

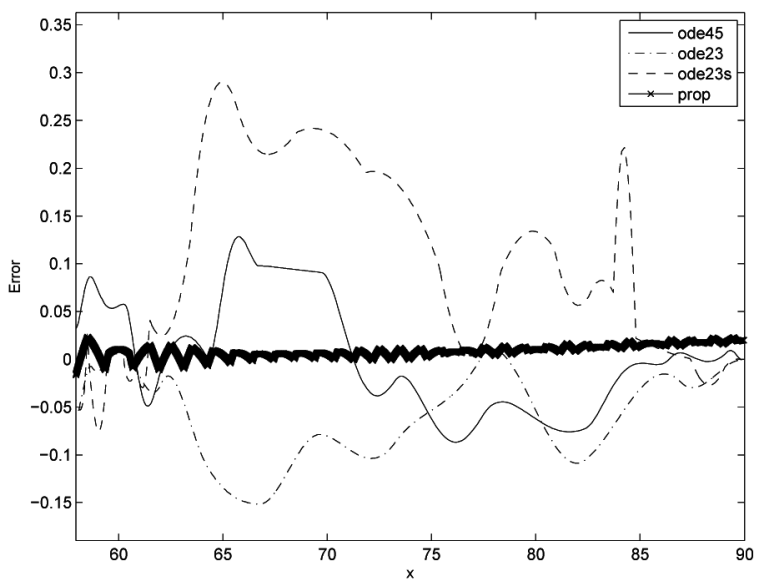

(d)

Fig. 5. Flowline computation examples over chirp images. We present results from computing a single flowline over chirp images $I\left(x_{1}, x_{2}\right)=\cos \phi\left(x_{1}, x_{2}\right)$ with $\phi\left(x_{1}, x_{2}\right)=a\left(x_{1}-x_{c 1}\right)^{2}+b\left(x-x_{c 2}\right)^{2}$ (see (57)). In all examples, we set $\left(x_{c 1}, x_{c 2}\right)=(50,50),\left(x_{1,0}, x_{2,0}\right)=(90,90)$ for all cases. (a) Flowline computation for $a=0.02, b=0.01$. (b) Error for $a=0.02, b=0.01$. (c) Flowline computation for $a=0.02, b=0.03$. (d) Error for $a=0.02, b=0.03$. For the proposed method, we used $\Delta t=0.01$. The proposed method exhibits significantly reduced error in all cases, at a reduced computational cost.

\subsection{FM Flowline and Ridge Computation in Fingerprint Images}

We recall from our discussion that image flowlines follow the direction of steepest ascent $\nabla \phi$. We thus expect that image flowlines originating from any point in the image, will follow a path of steepest ascent to a local maximum or local saddle point of the phase function. Based on these observations, we consider flowlines originating from the inside of the image, 10 pixels from the outside border, uniformly sampling the inside border every four pixels (see Fig. 6c).

For computing the IF vectors, we use the Teager-Kaiser operator with a constant-Q filterbank, as described in Section 2. For computing the IFGT, after sign-unwrapping described below, we convolve the IF vector field with derivatives of Gaussians with $\sigma=4.25$ of size $17 \times 17$ pixels.

To show how direction correction (see Fig. 4) affects flowline computation, compare the flowlines computed in Figs. $6 \mathrm{~d}$ and 6e. For these examples, we used the RungeKutta $(4,5)$ formula method (ode45(.) [48]). It is clear that the orientation discontinuity in the middle of the image has caused flowline computation to break down in the right part of Fig. 6d. By contrast, in Fig. 6e, flowlines converge toward the center of the image.

Even though direction correction appears to have worked here, recall that we cannot always produce continuous IF vector fields [1]. Furthermore, note that our direction correction algorithm cannot produce stable, converging flowlines. In our example in Fig. 6, the direction of all direction-corrected IF vectors was strongly affected by the fact that the upper left IF estimate was pointing toward the center of the image. If it was pointing outside the image, then the sign-unwrapped IF vectors would all point outwards (see, for example, all the flowlines computed from points in the lower right and bottom right of Fig. 6d).

The results for the proposed approach are shown in Fig. 6c. In comparison, we recall the results from the Runge-Kutta $(4,5)$ formula method (ode45(.), [48]) shown in Fig. 6e and a stiff solver (ode23s(.), [50]) shown in Fig. 6f. From the results, we can infer that the flowlines look similar from the three methods, except for the noisy flowline line segments seen in Fig. 6e in the upper-right to middle-right parts of the fingerprint. These noisy flowline segments are missing from Figs. $6 \mathrm{c}$ and $6 \mathrm{f}$. It also appears that the flowlines computed 

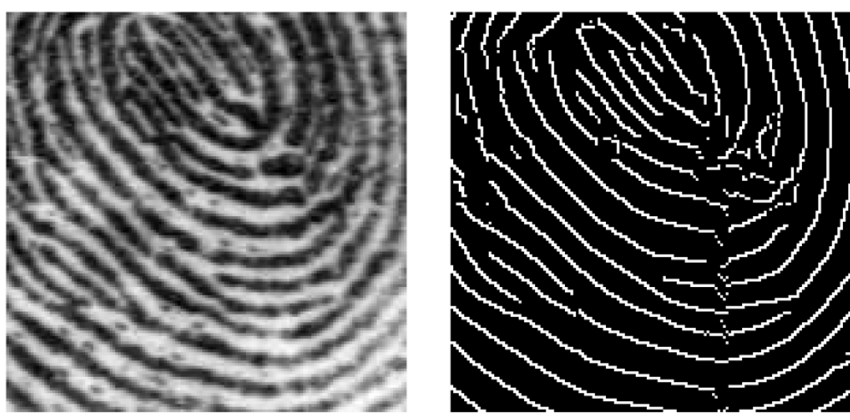

(a)

(b)
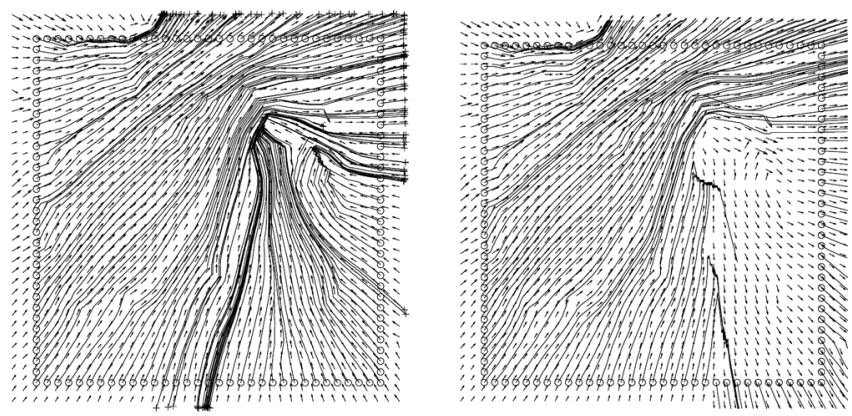

(c)

(d)

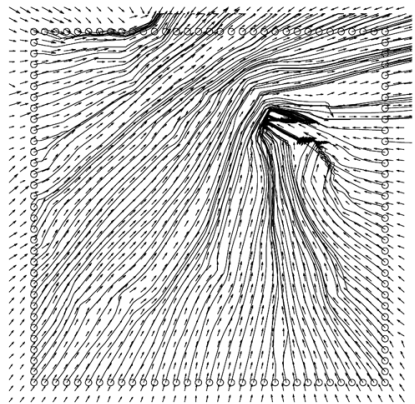

(e)

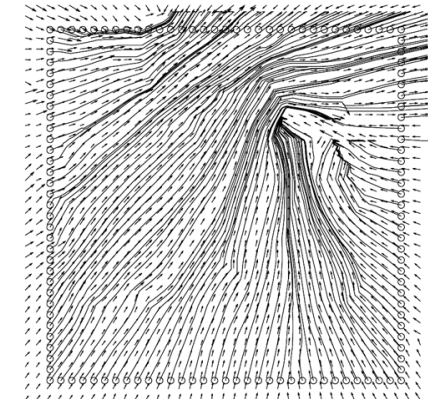

(f)

Fig. 6. Flowline and ridge extraction on a National Institute of Standards (NIST) fingerprint (second fingerprint). (a) Central part of the fingerprint. (b) Ridge detection on the FM image. Flowlines computed using: (c) the proposed method, (d) ode 45 (.) without direction correction, (e) ode 45 (.) with direction correction, and (f) ode23s (.) after direction correction.

with the proposed method (Fig. 6c) appear to cover the image plane more densely in the upper-right region. Thus, overall, especially given the well-known inaccurate estimates of stiff solvers (here, for ode $23 \mathrm{~s}(),.[50])$, we believe that the proposed method provides more accurate flowline estimates (also see the error curves in Fig. 5).

Using the same fingerprint, we also present ridgeextraction results in Fig. 6b. To compute the ridges, we detect image intensity minima along flowlines. To do this, at each pixel, we use a constant IF vector estimate to approximate the flowline through that pixel (extended to 32 pixels long). The sampled image intensity along the line segment is then filtered using a 1D Gabor bandpass filter to eliminate noise, and the filtered 1D signal is then used to determine image intensity minima. The image intensity minima are then

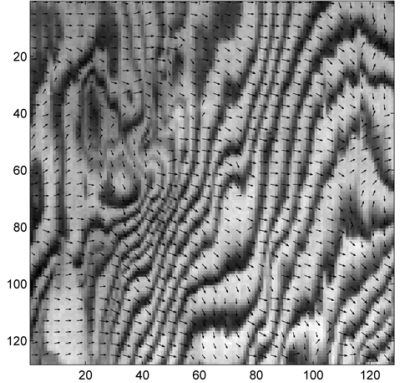

(a)

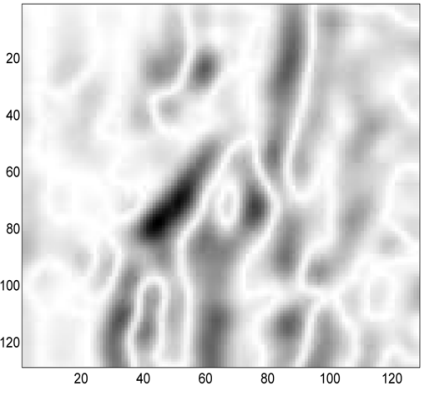

(b)

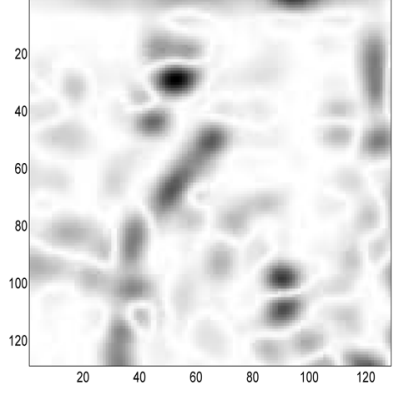

(c)
Fig. 7. Estimates of the divergence and curl for a woodgrain image. (a) Woodgrain image with instantaneous frequency estimates, (b) divergence, and (c) curl. For (a), the orientation vector magnitudes were plotted using $\log (1+100 * M)$, where $M$ denotes the vector magnitude. For (b) and (c), the absolute values of the divergence and the $z$-component of the curl vector are shown. For computing derivatives, we use convolution with derivatives of Gaussians. We note that dark regions correspond to high magnitude values, while white regions correspond to low (near-zero) values. Furthermore, the distinct white lines in (b) and (c) can be used to mark the regions where both the divergence and curl approach zero values. These lines can also be used to designate regions where there are sign changes in the divergence and the curl images.

mapped backed to their corresponding pixel coordinates and marked as ridge candidate points. All pixels that have been selected as ridge candidate points more than five times are designated as ridge points. This approach tends to reduce the number of false positive points (for ridge points). The extracted ridges in Fig. $6 \mathrm{~b}$ indicate that the underlying ridge structure has been successfully extracted using this approach, except possibly for the lack of horizontal connectivity in the middle-right of the image (due to the single-sided filterbank used in the demodulation).

\subsection{FM Analysis Example}

We demonstrate several concepts developed in this paper using the woodgrain image examples of Figs. 7 and 8. In the divergence image of Fig. 7b, points of significant compression in the woodgrain are captured by the dark islands throughout the image. By contrast, the curl measure is maximized when the woodgrain is locally characterized by significant curvatures. The curl of a gradient field is always zero. If a nonzero curl occurs, it is either due to 1) numerical measurement errors in the demodulation algorithm, or 2) actual discontinuities in the instantaneous frequency vector field. In the woodgrain image, when the grain makes very sharp corners, it is reasonable to think of the sharp corners as points where 


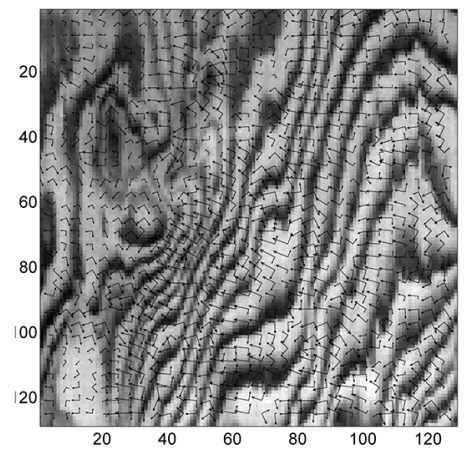

(a)

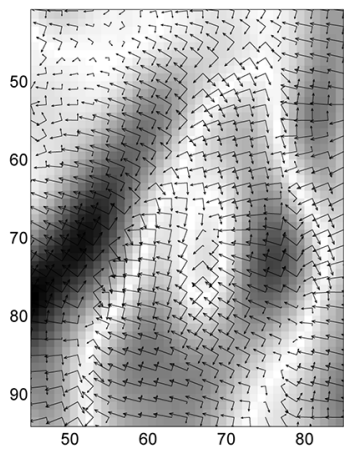

(c)

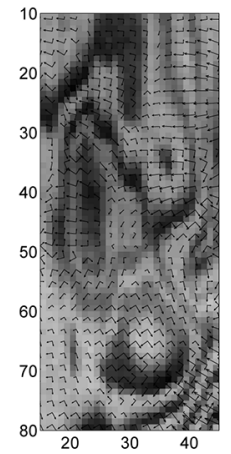

(b)

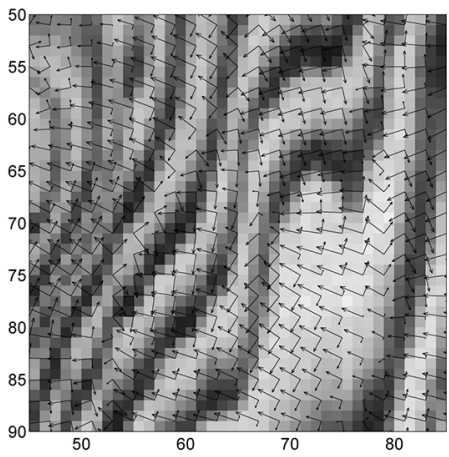

(d)
Fig. 8. Estimates of the IFGT components for a woodgrain image. (a) Woodgrain image with shape vectors estimates (see text), (b) zoom in on "ellipsoidal" deformations, (c) zoom in on divergence and shape vectors estimates, and (d) zoom in on image region corresponding to (c). In (a), one set of shape vectors are shown for every four pixels. In (b) and (c), one set of shape vectors is shown for every other pixel. Vector magnitudes were plotted using $\log (1+100 * M)$, where $M$ denotes the vector magnitude.

the instantaneous frequency vectors change orientation sharply, namely, where there are orientation discontinuities. The principal axes of deformation, described by "shape vectors" $\lambda_{i} \mathbf{e}_{i}$, are shown in Fig. 8. Assuming that the eigenvalues are of the same sign (refer to Fig. 2), compare the local woodgrain structure to that of a chirp image with quadratic phase. The longest "shape vector" defines the line direction of maximum woodgrain compression, while the shortest "shape vector" defines the line direction of minimum woodgrain compression (as in Figs. 8b and 8d). Note that the signs of the eigenvalues are not depicted in these figures and also note that FM is only a function of their magnitude (see Lemma 1). The signs of the eigenvalues are also a function of the chosen IF direction since $-\nabla \varphi$ will have an IFGT trace that has opposite sign than the sign of of the IFGT for $\nabla \varphi$. Also, when the eigenvectors are of opposite sign, we have a saddle point of the phase (see Fig. 2), and the shape vectors are still aligned with the directions of minimum and maximum compression. Furthermore, we note that the due to averaging and downsampling, the shape vectors are used to characterize relatively large neighborhoods (especially for Fig. 8d).

Two good examples are shown in Fig. 8b. In the middleleft of the image (near row 45, column 20), for the elongated ellipsoidal chirp-like deformations, the dominant shape vector $\lambda_{1} e_{1}$ is aligned with the horizontal direction, indicating substantial grain compression along this line,

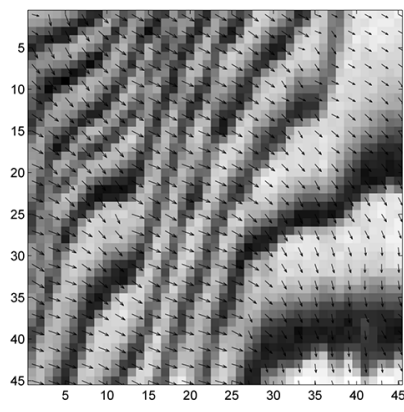

(a)

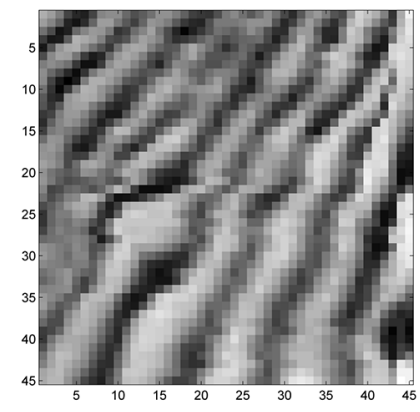

(b)
Fig. 9. An AM-FM harmonic image example, showing that a woodgrain texture can be approximated by a sinusoidal image that is coordinate transformed through a frequency modulation process. We have (a) the extracted woodgrain image with instantaneous frequency estimates. Every other instantaneous frequency vector is shown. Each vector magnitude $M$ is rescaled using $\log (1+100 * M)$. (b) The original image resampled in the inverse coordinate system (see text).

while a short $\lambda_{2} e_{2}$ correctly indicates smaller compression in the orthogonal direction. In the same image, in the lowerright image (near row 65, column 35), for the circular chirplike deformations, the shape vectors $\lambda_{1} e_{1}, \lambda_{2} e_{2}$ are of similar length, as expected. Similarly, in Fig. 8d, there are long shape vectors in the line-directions of maximal grain compression. Here, we use the term line-direction to refer to lines that extend in both the $\lambda_{i} e_{i}$ and $-\lambda_{i} e_{i}$ directions. In Fig. $8 \mathrm{c}$, the shape vectors are overlaid on the absolute value of the divergence image (which is equal to the sum of the eigenvalues). In general, long shape vectors are characterized by dark regions indicating that the eigenvalues may share the same sign. Note that the signs of the eigenvalues cannot be uniquely determined since both $\cos \phi$ and $\cos (-\phi)$ represent the same function.

We have thus developed several FM measures that allow us to quantify changes in the local spatial-frequency content. We believe that such FM measures can serve several important purposes in image analysis. At a very basic level, the discussion motivates the use of continuous-scale methods in image processing. By continuous-scale, we suggest that resolution requirements in image analysis vary continuously in space, as quantified by the FM measures presented here. As examples, we note that the eigenvalues provide measures of spatial-scale changes, while the eigenvectors also provide the directions of such changes. The divergence gives us a measure of scale-changes within the neighborhood, while the curl can be used to detect image singularities.

\subsection{FM-Based Coordinate System}

In this simple example, we selected the lower-middle portion of the woodgrain image of Fig. 8a. We see that the IF is never zero in the subimage of Fig. 8a. From our discussion on bifurcation points in Section 4.3, we can thus expect that a coordinate transformation must exist that can transform this subimage to a fixed frequency sinusoid. To compute such a coordinate transformation, we note that from a careful inspection of the IF vector field in Fig. 9a, we see that each point in the interior of the subimage can be reached by a flowline starting at the boundary of the subimage. We consider the simple, separable coordinate system, in terms of the vertical and horizontal components of the IF, starting from the boundary of the subimage: 


$$
s_{1}(x, y) \equiv \int_{x_{0}}^{x}\left|\frac{\partial \phi}{\partial x}(\tau, y)\right| d \tau
$$

and

$$
s_{2}(x, y) \equiv \int_{y_{0}}^{y}\left|\frac{\partial \phi}{\partial y}(x, \tau)\right| d \tau .
$$

We note that along each coordinate axis, (58) and (59) measure the total phase increase. Thus, in the new coordinate system of $s_{1}$ and $s_{2}$, we expect that the phase would appear to be changing at a more uniform rate than its rate of change in the original subimage. To check the validity of this claim, we invert the curvilinear coordinate system as demonstrated in Fig. 9b. From Fig. 9b, we note that this simple approximation appears to hold.

\section{Conclusion and Future Work}

We have developed a theory and have shown some applications of multidimensional frequency modulation models for image analysis. We believe that a wide variety of future image and video processing applications can be built on the theory presented here.

A number of new local texture measures were proposed for characterizing and understanding local spatial frequency content. At a more global level, the ODEs suggest themselves in the computation of coordinate systems that can be used to transform complex textured patterns to simple harmonics.

There is a wide range of applications, ranging from new image segmentation methods to image and video coding, that can benefit from the use of these new spatial frequency content measures and the ODES of frequency modulation. We have shown promising results on image segmentation (ridge extraction) and on the computation of curvilinear coordinate systems. In future work, it will be quite natural to explore global coordinate systems for developing image and video processing methods over well-suited, curvilinear coordinate systems defined in terms of multidimensional frequency modulation. We are currently investigating the use of spatial frequency content for scalable image and video coding applications, where we use the local spatial frequency content to control the number of nodes in globally defined, image-adaptive mesh systems.

\section{ApPendix A}

Proof of Lemma 1. We have:

$$
\begin{aligned}
\frac{\|d \mathbf{O}\|^{2}}{\|d \mathbf{x}\|^{2}} & =\frac{d \mathbf{O}^{T} d \mathbf{O}}{d \mathbf{x}^{T} d \mathbf{x}} \\
& =\frac{d \mathbf{x}^{T} \mathbf{F}^{T} \mathbf{F} d \mathbf{x}}{d \mathbf{x}^{T} d \mathbf{x}} \\
& =\frac{d \mathbf{x}^{T}\left(\mathbf{E} \boldsymbol{\Lambda} \mathbf{E}^{T}\right)\left(\mathbf{E} \Lambda \mathbf{E}^{T}\right) d \mathbf{x}}{d \mathbf{x}^{T} d \mathbf{x}} \\
& =\frac{d \mathbf{x}^{T}\left(\mathbf{E} \Lambda^{2} \mathbf{E}^{T}\right) d \mathbf{x}}{d \mathbf{x}^{T} d \mathbf{x}} .
\end{aligned}
$$

Going from (62) to (63) follows from the orthogonality of the eigenvectors: $\mathbf{E}^{T} \mathbf{E}=\mathbf{I}$. We recognize (63) as a Rayleigh Quotient for which (see [42, p. 111, (5)):

$$
\lambda_{1}^{2} \geq \frac{d \mathbf{x}^{T}\left(\mathbf{E} \Lambda^{2} \mathbf{E}^{T}\right) d \mathbf{x}}{d \mathbf{x}^{T} d \mathbf{x}} \geq \lambda_{2}^{2}, \quad \lambda_{1}^{2}>\lambda_{2}^{2}
$$

which, using (60), can be rewritten as:

$$
\lambda_{1}^{2} \geq \frac{\|d \mathbf{O}\|^{2}}{\|d \mathbf{x}\|^{2}} \geq \lambda_{2}^{2} .
$$

After multiplying each term by $\|d \mathbf{x}\|^{2}$, and taking square roots, (7) follows: Now, let $d \mathbf{x}=c\left[e_{1,1}, e_{1,2}\right]^{T}$, where $c$ is a scalar constant and $\left[e_{1,1}, e_{1,2}\right]^{T}$ denotes the first eigenvector. Using the orthonormality of the eigenvectors, evaluate (63) to get equality for the upper bound:

$$
\begin{aligned}
\frac{\|d \mathbf{O}\|^{2}}{\|d \mathbf{x}\|^{2}} & =\frac{d \mathbf{x}^{T}\left(\mathbf{E} \Lambda^{2} \mathbf{E}^{T}\right) d \mathbf{x}}{d \mathbf{x}^{T} d \mathbf{x}} \\
& =\frac{c \cdot 1 \cdot \lambda_{1}^{2} \cdot 1 \cdot c}{c^{2}} \\
& =\lambda_{1}^{2} .
\end{aligned}
$$

Equality for the lower bound for the second eigenvector follows similarly.

\section{APPENDIX B}

\section{ODE Proof for MULtidimensional FM}

Proof of Theorem 1 for general FM ODEs. From (13), it follows immediately that the flowline is given by (14).

For the instantaneous frequency vector along the flowline, we begin by differentiating the instantaneous frequency vector $\mathbf{O}(\mathbf{x}(t))$ with respect to time:

$$
\left[\begin{array}{l}
d O_{1} / d t \\
d O_{2} / d t
\end{array}\right]=\left[\begin{array}{ll}
\partial O_{1} / \partial x_{1} & \partial O_{1} / \partial x_{2} \\
\partial O_{2} / \partial x_{1} & \partial O_{2} / \partial x_{2}
\end{array}\right]\left[\begin{array}{l}
d x_{1} / d t \\
d x_{2} / d t
\end{array}\right]
$$

Next, substitute (13) in (69) to obtain:

$$
\frac{d \mathbf{O}(\mathbf{x}(t))}{d t}=\left[\begin{array}{ll}
\partial O_{1} / \partial x_{1} & \partial O_{1} / \partial x_{2} \\
\partial O_{2} / \partial x_{1} & \partial O_{2} / \partial x_{2}
\end{array}\right] \mathbf{O}(\mathbf{x}(t)) .
$$

Using the definition of the IFGT

$$
\frac{d \mathbf{O}}{d t}(\mathbf{x}(t))=\mathbf{F}(\mathbf{x}(t)) \mathbf{O}(\mathbf{x}(t))
$$

which immediately gives (15).

For the phase, differentiate $\phi(\mathbf{x}(t))$ with respect to time

$$
\frac{d \phi(\mathbf{x}(t))}{d t}=\left[\begin{array}{ll}
\partial \phi / \partial x_{1} & \partial \phi / \partial x_{2}
\end{array}\right]\left[\begin{array}{l}
d x_{1} / d t \\
d x_{2} / d t
\end{array}\right] .
$$

Substitute (13) into (72) to get

$$
\frac{d \phi(\mathbf{x}(t))}{d t}=\nabla \phi(\mathbf{x}(t)) \cdot \mathbf{O}(\mathbf{x}(t)) .
$$

By applying the definition of the instantaneous frequency $(\mathbf{O}=\nabla \phi)$, it follows that

$$
\frac{d \phi}{d t}(\mathbf{x}(t))=\|\mathbf{O}(\mathbf{x}(t))\|^{2}
$$

which yields the general expression given in (16). 


\section{APPENDIX C}

\section{Diagonalization of the ODEs and Phase SEPARABILITY}

Proof of Theorem 2. Using the linearity property [42, p. 161]:

$$
\frac{d \mathbf{z}}{d t}=\frac{d \mathbf{E}^{T}}{d t} \mathbf{x}+\mathbf{E}^{T} \frac{d \mathbf{x}}{d t}
$$

Since $\mathbf{U}$ is unitary, we get the norm of the error:

$$
\begin{aligned}
\left\|\varepsilon_{1}\right\| & =\left\|\frac{d \mathbf{z}}{d t}-\mathbf{E}^{T} \frac{d \mathbf{x}}{d t}\right\| \\
& =\left\|\frac{d \mathbf{E}^{T}}{d t} \mathbf{x}\right\| \\
& =\left\|\mathbf{U}_{1} \frac{d \theta}{d t} \mathbf{x}\right\| \\
& =\left|\frac{d \theta}{d t}\right|\|\mathbf{x}\| .
\end{aligned}
$$

Next, we differentiate (75) to get:

$$
\frac{d^{2} \mathbf{z}}{d t^{2}}=\frac{d^{2} \mathbf{E}^{T}}{d t^{2}} \mathbf{x}+2 \frac{d \mathbf{E}^{T}}{d t} \frac{d \mathbf{x}}{d t}+\mathbf{E}^{T} \frac{d^{2} \mathbf{x}}{d t^{2}} .
$$

Using (80), for the second error bound we get:

$$
\begin{aligned}
\left\|\varepsilon_{2}\right\| & =\left\|\frac{d^{2} \mathbf{z}}{d t^{2}}-\mathbf{E}^{T} \frac{d^{2} \mathbf{x}}{d t}\right\| \\
& =\left\|\frac{d^{2} \mathbf{E}^{T}}{d t^{2}} \mathbf{x}+2 \frac{d \mathbf{E}^{T}}{d t} \frac{d \mathbf{x}}{d t}\right\| .
\end{aligned}
$$

Next, we separate the terms inside the norm of (82) to get (see [42, p. 162]):

$$
\left\|\varepsilon_{2}\right\| \leq\left\|\frac{d^{2} \mathbf{E}^{T}}{d t^{2}} \mathbf{x}\right\|+2\left\|\frac{d \mathbf{E}^{T}}{d t} \frac{d \mathbf{x}}{d t}\right\| .
$$

We then use (45) to get

$$
\begin{aligned}
\left\|\varepsilon_{2}\right\| & \leq\left\|\mathbf{U}_{2}\left(\frac{d \theta}{d t}\right)^{2} \mathbf{x}\right\|+\left\|\mathbf{U}_{1} \frac{d^{2} \theta}{d t^{2}} \mathbf{x}\right\|+2\left\|\mid \mathbf{U}_{1} \frac{d \theta}{d t} \frac{d \mathbf{x}}{d t}\right\| \\
& \leq\left(\frac{d \theta}{d t}\right)^{2}\|\mathbf{x}\|+\left|\frac{d^{2} \theta}{d t^{2}}\right|\|\mathbf{x}\|+2\left|\frac{d \theta}{d t}\right|\|\mathbf{O}(\mathbf{x}(t))\| .
\end{aligned}
$$

Proof of Lemma 2 on Phase Separability. We begin with the expressions for the instantaneous frequency components. From [47, p, 21], the solution to (51) is given by:

$$
\begin{aligned}
& \tilde{O}_{1}(\mathbf{z}(t)) \approx a_{1} \exp \left(\int^{t} \tilde{\lambda}_{1}(\mathbf{z}(\tau)) d \tau\right), \\
& \tilde{O}_{2}(\mathbf{z}(t)) \approx a_{2} \exp \left(\int^{t} \tilde{\lambda}_{2}(\mathbf{z}(\tau)) d \tau\right) .
\end{aligned}
$$

Under the eigenvalue separability assumption, it is clear that we get (55) from (86) and (87).

Now, let $\mathbf{z}$ be any point in the interior of $S$. Then, by assumption, we can write (using the phase ODE (16)):

$$
\tilde{\phi}(\mathbf{z}(t))=\tilde{\phi}\left(\mathbf{z}_{0}\right)+\int_{0}^{t}\|\tilde{\mathbf{O}}(\mathbf{z}(\tau))\|^{2} d \tau
$$

where $\mathbf{z}_{0}$ is a point on the boundary of $S$. It is clear that the boundary phase value may always be expressed as a function of a single coordinate $\left(z_{1}\right.$ or $\left.z_{2}\right)$ :

$\tilde{\phi}\left(\mathbf{z}_{0}\right)= \begin{cases}\tilde{\phi}\left(z_{1}, z_{2, \text { min }}\right), & \text { if } \mathbf{z}_{0} \in\left[z_{1, \min }, z_{1, \max }\right] \times\left\{z_{2, \min }\right\} ; \\ \tilde{\phi}\left(z_{1}, z_{2, \max }\right), & \text { if } \mathbf{z}_{0} \in\left[z_{1, \min }, z_{1, \max }\right] \times\left\{z_{2, \max }\right\} ; \\ \tilde{\phi}\left(z_{1, \min }, z_{2}\right), & \text { if } \mathbf{z}_{0} \in\left\{z_{1, \min }\right\} \times\left[z_{2, \min }, z_{2, \max }\right] ; \\ \tilde{\phi}\left(z_{1, \max }, z_{2}\right), & \text { if } \mathbf{z}_{0} \in\left\{z_{1, \max }\right\} \times\left[z_{2, \min }, z_{2, \max }\right] .\end{cases}$

Next, we substitute (55) into (88) to get:

$$
\tilde{\phi}(\mathbf{z}(t)) \approx \tilde{\phi}\left(\mathbf{z}_{0}\right)+\int_{0}^{t}\left|\tilde{O}_{1}\left(z_{1}(\tau)\right)\right|^{2} d \tau+\int_{0}^{t}\left|\tilde{O}_{2}\left(z_{2}(\tau)\right)\right|^{2} d \tau
$$

From (90), we directly get (54).

\section{REFERENCES}

[1] R. Penrose, "The Topology of Ridge Systems," Annals of Human Genetics, vol. 42, pp. 435-444, 1979.

[2] J.J. Koenderink and A.J. van Doorn, "Local Features of Smooth Shapes: Ridges and Courses," SPIE: Proc. Geometric Methods in Computer Vision II, vol. 2031, pp. 2-13, 1993.

[3] A.V. Oppenheim and J.S. Lim, "The Importance of Phase in Signals," Proc. IEEE, vol. 69, pp. 529-541, May 1981.

[4] D.J. Fleet and A.D. Jepson, "Computation of Component Image Velocity from Local Phase Information," Int'l J. Computer Vision, vol. 5, no. 1, pp. 77-104, 1990.

[5] J.F. Kaiser, "On a Simple Algorithm to Calculate The 'Energy' of a Signal," Proc. IEEE Conf. Acoustics, Speech, and Signal Processing, pp. 381-384, Apr. 1990.

[6] P. Maragos, J.F. Kaiser, and T.F. Quatieri, "On Amplitude and Frequency Demodulation Using Energy Operators," IEEE Trans. Signal Processing, vol. 41, no. 4, pp. 1532-1550, Apr. 1993.

[7] P. Maragos, J.F. Kaiser, and T.F. Quatieri, "Energy Separation in Signal Modulations with Applications to Speech Analysis," IEEE Trans. Signal Processing, vol. 41, no. 10, pp. 3024-3051, Oct. 1993.

[8] A. Bovik, P. Maragos, and T. Quatieri, "AM-FM Energy Detection and Separation in Noise Using Multiband Energy Operators," IEEE Trans. Signal Processing, vol. 41, no. 12, pp. 3245-3265, Dec. 1993.

[9] G. Girolami and D. Vakman, "Instantaneous Frequency Estimation and Measurement: A Quasi-Local Method," Measurement Science Technology, vol. 13, pp. 909-917, June 2002.

[10] A. Bovik, N. Gopal, T. Emmoth, and A. Restrepo, "Localized Measurement of Emergent Image Frequencies by Gabor Wavelets," IEEE Trans. Information Theory, vol. 38, no. 3, pp. 691-712, Mar. 1992.

[11] P. Maragos and A. Bovik, "Image Amplitude and Frequency Demodulation Using Multidimensional Energy Separation," J. Optical Soc. Am., vol. 12, no. 9, pp. 1867-1876, Sept. 1995.

[12] B. Santhanam and P. Maragos, "Multicomponent AM-FM Demodulation Via Periodicity-Based Algebraic Separation and Energy-Based Demodulation," IEEE Trans. Comm., vol. 48, no. 3, pp. 473-490, Mar. 2000.

[13] D. Dimitriadis and P. Maragos, "An Improved Energy Demodulation Algorithm Using Splines," Proc. IEEE Conf. Acoustics, Speech, and Signal Processing, May 2001.

[14] D. Dimitriadis and P. Maragos, "Robust Energy Demodulation Based on Continuous Models with Application to Speech Recognition," Proc. European Conf. Speech Comm. and Technology, Sept. 2003.

[15] B. Santhanam, "Generalized Energy Demodulation for Large Frequency Deviations and Wideband Signals," IEEE Signal Processing Letters, vol. 11, no. 3, pp. 341-344, Mar. 2004.

[16] J.P. Havlicek, D.S. Harding, and A.C. Bovik, "Multidimensional Quasi-Eigenfunction Approximations and Multicomponent AMFM Models," IEEE Trans. Image Processing, vol. 9, no. 2, pp. 227242, Feb. 2000

[17] J.P. Havlicek, "AM-FM Image Models," PhD dissertation, The Univ. of Texas at Austin, 1996. 
[18] R.A. Baxter and T.F. Quatieri, "Shunting Networks for Multi-Band AM-FM Decomposition," Proc. IEEE Workshop Applications of Signal Processing to Audio and Acoustics, pp. 227-230, Oct. 1999.

[19] M.R. Morelande and A.M. Zoubir, "Model Selection of Random Amplitude Polynomial Phase Signals," IEEE Trans. Signal Processing, vol. 50, no. 3, pp. 578-589, Mar. 2002.

[20] S.L. Hahn, "The Analytic, Quaternionic and Monogenic 2D Complex Delta Distributions," Technical Report 3, Inst. of Radioelectronics, Nowowiejska, Warsaw Univ. of Technology, Apr. 2002.

[21] T. Bülow and G. Sommer, "Hypercomplex Signals-A Novel Extension of the Analytic Signal to the Multidimensional Case," IEEE Trans. Signal Processing, vol. 49, no. 11, pp. 2844-2852, Nov. 2001

[22] U. Köthe and M. Felsberg, "Riesz-Transforms vs. Derivatives: On the Relationship between the Boundary Tensor and the Energy Tensor," Proc. Scale Space and PDE Methods in Computer Vision, R. Kimmel, N. Sochen, and J. Weickert, eds., pp. 179-191, 2005.

[23] H. Knutsson, C.-F. Westin, and G.H. Granlund, "Local Multiscale Frequency and Bandwidth Estimation," Proc. IEEE Int'l Conf. Image Processing, pp. 36-40, Nov. 1994.

[24] K.G. Larkin, D.J. Bone, and M.A. Oldfield, "Natural Demodulation of Two-Dimensional Fringe Patterns. I. General Background of the Spiral Phase Quadrature Transform," J. Optical Soc. Am. A, vol. 18, no. 8, pp. 1862-1870, Aug. 2001.

[25] K.G. Larkin, "Natural Demodulation of Two-Dimensional Fringe Patterns. II. Stationary Phase Analysis of the Spiral Phase Quadrature Transform," J. Optical Soc. Am. A, vol. 18, no. 8, pp. 1871-1881, Aug. 2001

[26] K.G. Larkin, "Uniform Estimation of Orientation Using Local and Nonlocal 2D Energy Operators," Optics Express, vol. 13, no. 20, pp. 8097-8121, Oct. 2005.

[27] M. Felsberg and U. Köthe, "Get: The Connection between Monogenic Scale-Space and Gaussian Derivatives," Proc. ScaleSpace Conf., 2005

[28] M. Felsberg and G. Sommer, "The Monogenic Signal," IEEE Trans. Signal Processing, vol. 49, no. 12, pp. 3136-3144, Dec. 2001.

[29] A.C. Bovik, J.P. Havlicek, M.D. Desai, and D.S. Harding, "Limits on Discrete Modulated Signals," IEEE Trans. Signal Processing, vol. 45, no. 4, pp. 867-879, Apr. 1997

[30] W.-C. Pai and P.C. Doerschuk, "Statistical AM-FM Models, Extended Kalman Filter Demodulation, Cramer-Rao Bounds, and Speech Analysis," IEEE Trans. Signal Processing, vol. 48, no. 8, pp. 2300-2313, Aug. 2000.

[31] B.J. Super and A.C. Bovik, "Shape from Texture Using Local Spectral Moments," IEEE Trans. Pattern Analysis and Machine Intelligence, vol. 17, no. 4, pp. 333-343, Apr. 1995.

[32] S.T. Acton, D.P. Mukherjee, J.P. Havlicek, and A.C. Bovik, "Oriented Texture Completion by AM-FM Reaction-Diffusion," IEEE Trans. Image Processing, vol. 10, no. 6, pp. 885-896, June 2001.

[33] M. Pattichis, G. Panayi, A. Bovik, and H. Shun-Pin, "Fingerprint Classification Using and AM-FM Model," IEEE Trans. Image Processing, vol. 10, no. 6, pp. 951-954, June 2001.

[34] J. Havlicek, J. Tang, S. Acton, R. Antonucci, and F. Quandji, "Modulation Domain Texture Retrieval for CBIR in Digital Libraries," Proc. 37th IEEE Asilomar Conf. Signals, Systems, and Computers, Nov. 2003.

[35] M. Pattichis, C. Pattichis, M. Avraam, A. Bovik, and K. Kyriakou, "AM-FM Texture Segmentation in Electron Microscopic Muscle Imaging," IEEE Trans. Medical Imaging, vol. 19, no. 12, pp. 12531258, Dec. 2000.

[36] T. Yap, J. Havlicek, and V. DeBrunner, "Bayesian Segmentation of AM-FM Texture Images," Proc. 35th Asilomar Conf. Signals, Systems, and Computers, vol. 2, pp. 1156-1160, Nov. 2001.

[37] N. Ray, J. Havlicek, S.T. Acton, and M.S. Pattichis, "Active Contour Segmentation Guided by AM-FM Dominant Component Analysis," Proc. IEEE Int'l Conf. Image Processing, pp. 78-81, 2001.

[38] P.V. Rodriguez, M. Pattichis, and M. Goens, "M-Mode Echocardiography Image and Video Segmentation Based on AM-FM Demodulation Techniques," Proc. 25th Int'l Conf. IEEE Eng. Medicine and Biology Soc., vol. 2, pp. 1176-1179, Sept. 2003.

[39] G. Strang, Linear Algebra and Its Applications, third ed. Harcourt Brace Jovanovich, 1988

[40] R. Aris, Vectors, Tensors, and the Basic Equations of Fluid Mechanics. Prentice Hall, 1962.

[41] J.E. Marsden and A.J. Tromba, Vector Calculus, second ed. Freeman, 1981.

[42] R. Bellman, Introduction to Matrix Analysis. SIAM, 1995.
[43] V. Guillemin and A. Pollack, Differential Topology. Prentice-Hall, 1974.

[44] L.R. Nackman, "Two-Dimensional Critical Point Configuration," IEEE Trans. Pattern Analysis and Machine Intelligence, vol. 6, no. 4, pp. 442-250, Apr. 1984.

[45] M. Morse and S.S. Cairns, Critical Point Theory, second ed. Wiley, 1969.

[46] C. Steger, "Subpixel-Precise Extraction of Watersheds," Proc. Int'l Conf. Computer Vision, vol. II, pp. 884-890, 1999.

[47] W.E. Boyce and R.C. Diprima, Elementary Differential Equations and Boundary Value Problems, fourth ed. Wiley, 1986.

[48] J.R. Dormand and P.J. Prince, "A Family of Embedded RungeKutta Formulae," J. Computer Applied Math., vol. 6, pp. 19-26, 1980.

[49] P. Bogacki and L.F. Shampine, "A 3(2) Pair of Runge-Kutta Formulas," Applied Math. Letters, vol. 2, pp. 1-9, 1989.

[50] L.F. Shampine and M.W. Reichelt, "The Matlab ODE Suite," SIAM J. Scientific Computing, vol. 18, pp. 1-22, 1997.

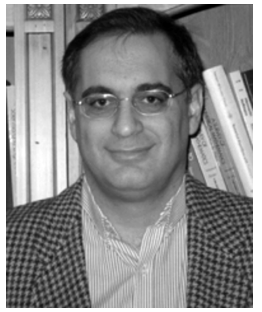

Marios S. Pattichis received the BSc degree (high honors and special honors) in computer sciences in 1991, the BA degree (high honors) in mathematics in 1991, the MS degree in engineering in 1993, and the $\mathrm{PhD}$ degree in computer engineering in 1998, all from the University of Texas at Austin. Since 2000, he has been an assistant professor with the Department of Electrical and Computer Engineering at the University of New Mexico (UNM), Albuquerque. Since 2005, he has also also an adjunct assistant professor in Radiology with the Department of Radiology at UNM. His research is in the areas of medical image and video processing, digital image and video models, radar image processing, SIMD, and reconfigurable computer architecture applications. He is an associate editor for Pattern Recognition and the general chair of the 2008 IEEE Southwest Symposium on Image Analysis and Interpretation, to be held in Santa Fe, New Mexico. At UNM, he received the 2003 ECE distinguished teaching award and the 2006 School of Engineering Harrison faculty recognition award. He is a senior member of the IEEE.

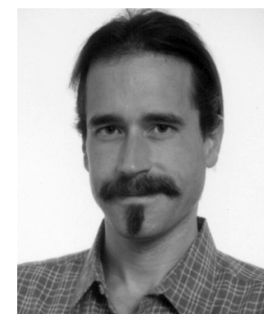

Alan C. Bovik is the Curry/Cullen Trust Endowed Chair Professor at The University of Texas at Austin, where he is the director of the Laboratory for Image and Video Engineering (LIVE). His research interests include image and video processing, computational vision, digital microscopy, and modeling of biological visual perception. He has published more than 450 technical articles in these areas and holds two US patents. He is the author of The Handbook of Image and Video Processing, second edition (Elsevier Academic Press, 2005) and Modern Image Quality Assessment (Morgan \& Claypool, 2006). Dr. Bovik has received the Technical Achievement Award from the IEEE Signal Processing Society in 2005, the Distinguished Lecturer Award from the IEEE Signal Processing Society in 2000, the IEEE Signal Processing Society Meritorious Service Award in 1998, the IEEE Third Millennium Medal in 2000, and is a twotime Honorable Mention winner of the international Pattern Recognition Society Award for Outstanding Contribution (1988 and 1993). He is a fellow of the IEEE and has been involved in numerous professional society activities, including: board of governors, IEEE Signal Processing Society, 1996-1998; editor-in-chief, IEEE Transactions on Image Processing, 1996-2002; editorial board, The Proceedings of the IEEE, 1998-2004; series editor for Image, Video, and Multimedia Processing, Morgan and Claypool Publishing Company, 2003-present; and Founding General Chairman, First IEEE International Conference on Image Processing, held in Austin, Texas, in November, 1994. Dr. Bovik is a registered professional engineer in the state of Texas and is a frequent consultant to legal, industrial, and academic institutions.

$\triangleright$ For more information on this or any other computing topic, please visit our Digital Library at www.computer.org/publications/dlib. 